\title{
GLOBAL SOLVABILITY OF A DISSIPATIVE FRÉMOND MODEL FOR SHAPE MEMORY ALLOYS. PART II: EXISTENCE
}

$\mathrm{BY}$

ELENA BONETTI

Dipartimento di Matematica "F. Casorati", Università di Pavia, Via Ferrata 1, 27100 Pavia, Italy

\begin{abstract}
The paper investigates an initial and boundary values problem which is derived from a dissipative Frémond model for shape memory alloys. Existence of a global solution for the abstract version of the evolution problem is proved by use of a semi-implicit time discretization scheme combined with an a priori estimates-passage to the limit procedure.
\end{abstract}

1. Introduction to the problem. In this paper we aim to prove a global existence result for a three dimensional dissipative model for shape memory proposed by Frémond. For a detailed presentation of the thermomechanical model and the derivation of a mathematical formulation we refer to [5] and references therein, where we have also proved uniqueness of the solution for the related initial and boundary values problem in the case when higher order quadratic terms are neglected in the energy balance equation.

Shape memory alloys are materials characterized by the possibility of recovering their original shape just by thermal means after mechanical deformations (cf. $[10,11,13.16]$ ). It is known that the phenomenon of shape memory behavior can be ascribed to a solidsolid phase transition between two different configurations of the metallic lattice, called austenite and martensite. In the macroscopic model proposed by Frémond, one variant of austenite and two variants of martensite are considered and it is assumed that they may coexist at each point. Hence, the model is written in terms of the absolute temperature $\theta$. the vector of small displacements $\mathbf{u}$, and two phase parameters $\left(\chi_{1}, \chi_{2}\right)$ linearly related to the volumetric fractions of the variants of martensite and austenite. In addition, the phase parameters are forced to fulfill an internal constraint forcing the volumetric fractions of the phases to assume only meaningful values, in the sense that no void or overlapping can occur. The constitutive equations of the Frémond model are derived by two functionals, the free energy and the pseudo-potential of dissipation (cf. $[12,13])$ for a diffusive phase transformation. Hence, a PDE's system is written in terms of the energy balance and the principle of virtual power written both for macroscopic movements. in a

Received December 27, 2001.

2000 Mathematics Subject Classification. Primary 35K85, 74Nxx, 35Q72.

E-mail address: bonetti@dimat.unipv.it 
quasi-stationary situation, and for microscopic velocities, which are related to the time derivatives of the phase parameters $\left(\chi_{1 t}, \chi_{2 t}\right)$.

Now, let us introduce the set of partial differential equations related to the model proposed by Frémond and the associated initial and boundary conditions. We consider a smooth bounded domain $\Omega \subseteq \mathbf{R}^{3}$ with $\Gamma=\partial \Omega=\Gamma_{0} \cup \Gamma_{1}$, the measure of $\Gamma_{0}$ being strictly positive, and describe the evolution of the thermomechanical system during a finite time interval $(0, T)$ and denote by $Q:=\Omega \times(0, T)$. Let us point out that we do not detail the physical meaning of the ingredients of the following mathematical formulation of the model for which we refer to [12] and [5], where the system is derived by the balance laws and the constitutive equations in terms of the free energy functional and the pseudopotential of dissipation. Here, for the sake of synthesis, the problem is directly written in the abstract framework of a Hilbert triplet $V \hookrightarrow H \hookrightarrow V^{\prime}$ with $H:=L^{2}(\Omega)$ and $V:=H^{1}(\Omega)$. We denote by $\langle\cdot, \cdot\rangle$ the duality pairing between $V^{\prime}$ and $V$ and identify as usual $H$ with its dual space $H^{\prime}$. Finally, by abuse of notation, we let $\|\cdot\|_{H}$ stand for the norm both in $H$ and $H^{3}$. We also introduce the Hilbert space

$$
\mathbf{W}:=\left\{\mathbf{v} \in V^{3}: \mathbf{v}=0 \text { on } \Gamma_{0} \text { and } \operatorname{div} \mathbf{v} \in V\right\},
$$

endowed with the norm

$$
\|\mathbf{u}\|_{\mathbf{W}}^{2}=\int_{\Omega}|\nabla \operatorname{div} \mathbf{v}|^{2}+\sum_{i, j=1}^{3} \int_{\Omega}\left(\partial_{x_{i}} v_{j}\right)^{2} \quad \mathbf{v}=\left(v_{1}, v_{2}, v_{3}\right) \in \mathbf{W} .
$$

In addition, let us specify a bilinear continuous symmetric form on $\mathbf{W} \times \mathbf{W}$ by

$$
a(u, v)=\nu \int_{\Omega} \nabla \operatorname{div} \mathbf{u} \cdot \nabla \operatorname{div} \mathbf{v}+\lambda_{L} \int_{\Omega} \operatorname{div} \mathbf{u} \operatorname{div} \mathbf{v}+2 \mu_{L} \sum_{i, j=1}^{3} \int_{\Omega} \varepsilon_{i j}(\mathbf{u}) \varepsilon_{i j}(\mathbf{v}),
$$

$\lambda_{L}$ and $\mu_{L}$ being the Lamé constants and $\nu>0$. Notice that by the Korn's inequality we can deduce that it is $\mathbf{W}$-elliptic, i.e.,

$$
a(\mathbf{u}, \mathbf{u}) \geq C\|\mathbf{u}\|_{\mathbf{W}}^{2}, \quad C>0,
$$

as well as it is a standard matter to verify that the following inequality holds (cf. [8]):

$$
a(\mathbf{u}, \mathbf{u}) \geq \nu\|\operatorname{div} \mathbf{v}\|_{H}^{2}+\left(\lambda_{L}+2 \mu_{L} / 3\right)\|\operatorname{div} \mathbf{v}\|_{H}^{2} .
$$

Hence, we introduce the operators

$$
\begin{aligned}
& A: V \rightarrow V^{\prime}, \quad\langle A u, v\rangle=\int_{\Omega} \nabla u \cdot \nabla v \quad u, v \in V, \\
& \mathcal{H}: \mathbf{W} \rightarrow \mathbf{W}^{\prime}, \quad\langle\mathcal{H} \mathbf{u}, \mathbf{v}\rangle=a(\mathbf{u}, \mathbf{v}) \quad \mathbf{u}, \mathbf{v} \in \mathbf{W}, \\
& \mathcal{B}: H \rightarrow \mathbf{W}^{\prime}, \quad\langle\mathcal{B} h, v\rangle=\int_{\Omega} h \operatorname{div} \mathbf{u} \quad h \in H, \mathbf{u} \in \mathbf{W},
\end{aligned}
$$

and two functions $\mathcal{R}$ and $\mathcal{G}$, in $V^{\prime}$ and $\mathbf{W}^{\prime}$ respectively, collecting external thermomechanical forces. More precisely, we introduce an external rate of heat production $r$ and the heat flux $h$ through the boundary, whose contributions are collected by the function $\mathcal{R}$ in $V^{\prime}$ specified by

$$
\langle\mathcal{R}, v\rangle=\left\langle\mathcal{R}_{1}+\mathcal{R}_{2}, v\right\rangle=\int_{\Omega} r v+\int_{\Gamma} h v_{\mid \Gamma} \quad v \in V
$$


Analogously, letting $\mathbf{G}$ be an exterior volumic force acting on the body and $\mathbf{g}$ a traction applied on $\Gamma_{1}$, we define $\mathcal{G}$ in $\mathbf{W}^{\prime}$ as follows:

$$
\mathbf{w}^{\prime}\langle\mathcal{G}, \mathbf{w}\rangle_{\mathbf{w}}=\int_{\Omega} \mathbf{G} \cdot \mathbf{w}+\int_{\Gamma_{1}} \mathbf{g} \cdot \mathbf{w}_{\mid \Gamma} \quad \mathbf{w} \in \mathbf{W} .
$$

The constraint on $\left(\chi_{1}, \chi_{2}\right)$ is imposed by the presence of a subdifferential term in the equation governing the phases' dynamics, forcing the parameters to assume only physically meaningful values. Towards this aim, in our abstract framework, we can fix any bounded closed convex subset $K$ of $H^{2}$ such that $0 \in K$ and refer, i.e., to [5] for a precise form of $K$ derived by the model. Let us in particular observe that, by construction, there exists a positive constant $c_{K}$, depending on $K$, such that for any $\left(\gamma_{1}, \gamma_{2}\right) \in K$, there holds

$$
\left(\left|\gamma_{1}\right|^{2}+\left|\gamma_{2}\right|^{2}\right)^{1 / 2} \leq c_{K} \text { a.e. in } \Omega
$$

For the reader's convenience we also recall the definition of the indicator function $I_{K \cap V^{2}}$ of $K \cap V^{2}, I_{K \cap V^{2}}(y)=0$ if $y \in K \cap V^{2}$ and $I_{K \cap V^{2}}(y)=+\infty$ otherwise, and the subdifferential operator of $I_{K \cap V^{2}}$ from $V^{2}$ in $\left(V^{\prime}\right)^{2}$, i.e., (cf. [3])

$$
\begin{aligned}
& \left(\xi_{1}, \xi_{2}\right) \in\left(V^{\prime}\right)^{2} \text { belongs to } \partial I_{K, V}\left(\chi_{1}, \chi_{2}\right) \text { if and only if } \\
& \left(\chi_{1}, \chi_{2}\right) \in K \cap V^{2} \text { and } \sum_{i=1}^{2}\left\langle\xi_{i}, \gamma_{i}-\chi_{i}\right\rangle \leq 0 \forall\left(\gamma_{1}, \gamma_{2}\right) \in K \cap V^{2} .
\end{aligned}
$$

Next, we can formulate the abstract problem we aim to solve. Let us point out that for the sake of simplicity, we have fixed some strictly positive physical constants equal to 1 (see the complete model in [5, Sec. 2]).

Problem $P$. Find $\left(\theta, \mathbf{u}, \chi_{1}, \chi_{2}\right)$ satisfying the Cauchy conditions

$$
\begin{aligned}
\theta(0) & =\theta_{0}, \\
\chi_{i}(0) & =\chi_{i 0} \quad i=1,2,
\end{aligned}
$$

and fulfilling a.e. in $(0, T)$

$$
\begin{aligned}
& F\left(\theta, \mathbf{u}, \chi_{1}, \chi_{2}\right) \theta_{t}+A \theta=\mathcal{R}+\theta \lambda^{\prime}(\theta) \chi_{1 t}+\theta \alpha^{\prime}(\theta) \operatorname{div} \mathbf{u} \chi_{2 t} \\
& +\theta \alpha^{\prime}(\theta) \chi_{2} \operatorname{div} \mathbf{u}_{t}+\sum_{i=1}^{2}\left(\chi_{i t}\right)^{2} \text { in } V^{\prime} \\
& \left(\begin{array}{l}
\chi_{1 t} \\
\chi_{2 t}
\end{array}\right)+\left(\begin{array}{l}
A \chi_{1 t} \\
A \chi_{2 t}
\end{array}\right)+\left(\begin{array}{c}
A \chi_{1} \\
A \chi_{2}
\end{array}\right)+\partial I_{K, V}\left(\chi_{1}, \chi_{2}\right) \ni\left(\begin{array}{c}
-\lambda(\theta) \\
-\alpha(\theta) \operatorname{div} \mathbf{u}
\end{array}\right) \text { in }\left(V^{\prime}\right)^{2}, \\
& \mathcal{H} \mathbf{u}+\mathcal{B}\left(\alpha(\theta) \chi_{2}\right)=\mathcal{G} \text { in } \mathbf{W}^{\prime},
\end{aligned}
$$

where $F$ in (1.15) is specified by

$$
F\left(\theta, \mathbf{u}, \chi_{1}, \chi_{2}\right)=c_{s}-\theta \alpha^{\prime \prime}(\theta) \operatorname{div} \mathbf{u} \chi_{2}+\theta \lambda^{\prime \prime}(\theta)\left(1-\chi_{1}\right) .
$$

Let us make precise the ingredients of the above system (1.15)--(1.17) and (1.18). The constant $c_{s}$ is positive and stands for the heat capacity of the system, while the function $\lambda$ is related to the energy associated to the phase transition. It is assumed that $\lambda$ is a nondecreasing bounded function such that $\lambda\left(\theta_{*}\right)=0$, with $\theta_{*}$ being the critical transition 
temperature, and strictly increasing in a neighborhood of $\theta_{*}$. In addition, we require that $\lambda$ fulfills

$$
\begin{aligned}
& \lambda \in W^{2 . \infty}(\mathbf{R}) \cap C^{2}(\mathbf{R}), \\
& \|\lambda\|_{W^{2 . \infty}(\mathbf{R})}+\left|\lambda^{\prime}(\xi) \xi\right| \leq \tilde{c}_{\lambda}, \quad\left|\lambda^{\prime \prime}(\xi) \xi\right| \leq c_{\lambda}, \forall \xi \in \mathbf{R},
\end{aligned}
$$

for $\tilde{c}_{\lambda}, c_{\lambda}>0$. Note, in particular, the additional boundedness requirements in (1.19) with respect to the natural bound of the functions in $W^{2, \infty}$. Concerning the function $\alpha$, representing a thermal expansion coefficient, it is prescribed as a nonnegative bounded function, nonincreasing, and vanishing over the interval $\left(\theta_{c},+\infty\right), \theta_{c}>\theta_{*}$ being the so-called Curie temperature. More precisely, we let

$$
\begin{aligned}
& \alpha \in C^{2}(\mathbf{R}), \quad\left\{\xi \in \mathbf{R}: \alpha^{\prime}(\xi) \neq 0\right\} \subseteq\left[0, \theta_{c}\right], \\
& \left|\alpha^{\prime \prime}(\xi)\right| \leq c_{\alpha} \quad \forall \xi \in \mathbf{R}, \quad \forall \xi \in \mathbf{R} .
\end{aligned}
$$

As a consequence of (1.20) we have, in particular, that

$$
\left|\alpha^{\prime}(\xi)\right| \leq c_{\alpha} \theta_{c}, \quad\left|\xi \alpha^{\prime}(\xi)\right| \leq c_{\alpha} \theta_{c}^{2} .
$$

Moreover, let us point out that the above assumptions are physically consistent as it is justified in our first paper on this subject [5]. Finally, in order to prove an existence result for the Problem $P$ we have to set some compatibility conditions on the involved quantities, but for the sake of convenience we will specify them later. Nonetheless, as they regard $c_{\alpha}$ and $c_{\lambda}$, we can postulate in advance

$$
c_{\alpha} \text { and } c_{\lambda} \text { are sufficiently small. }
$$

REMARK 1.1. We point out at once that the variational inclusion governing the phases' dynamics (1.16) is written in the abstract setting of the $\left(V^{\prime}\right)^{2}-V^{2}$ duality pairing. Nonetheless, even if it cannot be written a.e. in $Q$, the model retains its physical consistence, since (1.16) forces the phases to attain only meaningful values, i.e., $\left(\chi_{1}, \chi_{2}\right) \in K$ (cf. Remark 3.2 and 3.3 in [5]).

Now, let us specify the hypotheses on the data of the above problem. We prescribe that

$$
\begin{gathered}
r \in L^{2}\left(0, T ; L^{2}(\Omega)\right), \\
h \in W^{1,1}\left(0, T ; L^{2}(\Gamma)\right), \\
\mathbf{G} \in H^{1}\left(0, T ; L^{2}(\Omega)^{3}\right), \\
\mathbf{g} \in H^{1}\left(0, T ; L^{2}\left(\Gamma_{1}\right)^{3}\right),
\end{gathered}
$$

so that we have (cf. (1.9) and (1.10))

$$
\begin{aligned}
& \mathcal{R}=\mathcal{R}_{1}+\mathcal{R}_{2} \in L^{2}(0, T ; H)+W^{1.1}\left(0, T ; V^{\prime}\right), \\
& \mathcal{G} \in H^{1}\left(0, T ; \mathbf{W}^{\prime}\right)
\end{aligned}
$$

Finally, we assume

$$
\begin{aligned}
& \theta_{0} \in H^{1}(\Omega), \\
& \left(\chi_{10}, \chi_{20}\right) \in H^{2}(\Omega)^{2} \cap K, \quad \partial_{n} \chi_{i 0}=0 \quad i=1,2 \text { a.e. on } \Gamma .
\end{aligned}
$$

Here is the precise formulation of the existence result. 
TheOREM 1.1. Let (1.19), (1.20), (1.22), (1.23)-(1.26) (i.e., (1.27)-(1.28)), and (1.29)(1.30) hold. Then, there exists a quadruple of functions $\left(\theta, \mathbf{u}, \chi_{1}, \chi_{2}\right)$ solving Problem $P$ and fulfilling

$$
\begin{aligned}
& \theta \in H^{1}(0, T ; H) \cap L^{\infty}(0, T ; V), \\
& \mathbf{u} \in H^{1}(0, T ; \mathbf{W}) \text { with } \operatorname{div} \mathbf{u} \in H^{1}\left(0, T ; H^{2}(\Omega)\right), \\
& \chi_{i} \in W^{1, \infty}(0, T ; V) \cap L^{\infty}\left(0, T ; H^{2}(\Omega)\right), \quad i=1,2 .
\end{aligned}
$$

REmark 1.2. Concerning the uniqueness of the solution to the Problem $P$, we recall that in [5] by use of a contracting argument we have proved it holds for a solution $\left(\theta, \mathbf{u},\left(\chi_{1}, \chi_{2}\right)\right)$ fulfilling $(1.31)-(1.33)$ in the case when all the quadratic dissipative terms in the energy balance are neglected.

2. Approximation of the problem. In order to achieve the proof of Theorem 1.1, we need to state a preliminary result concerning the well-posedness of Eq. (1.17), which is fairly standard in the theory of elliptic boundary value problems. Thus, we omit the detail and refer to [9] for the existence result and to [6] for the regularity statement, which mainly exploit the Lax-Milgram theorem and well-known regularity results on elliptic equations.

Lemma 2.1. Let $\hat{\theta}$ and $\hat{\chi}_{2}$ belong to $L^{2}(0, T ; H)$ and fulfill $\left|\hat{\chi}_{2}\right| \leq c_{K}$ a.c. in $Q$ (cf. (1.11)), $\alpha \in W^{1, \infty}(\mathbf{R})$, and $\mathcal{G} \in L^{\infty}\left(0, T ; \mathbf{W}^{\prime}\right)$. Then, there exists a unique

$$
\mathbf{u} \in L^{\infty}(0, T ; \mathbf{W})
$$

satisfying

$$
\mathcal{H} \mathbf{u}+\mathcal{B}\left(\alpha(\hat{\theta}) \hat{\chi}_{2}\right)=\mathcal{G} \text { in } \mathbf{W}^{\prime}
$$

a.e. in $(0, T)$. Moreover, the following inequality can be proved (by use of the Sobolev inclusion $\left.H^{2}(\Omega) \hookrightarrow L^{\infty}(\Omega)\right)$ :

$$
\|\operatorname{div} \mathbf{u}\|_{L^{\infty}(Q)} \leq c\|\operatorname{div} \mathbf{u}\|_{L^{\infty}\left(0, T ; H^{2}(\Omega)\right)} \leq c_{1},
$$

for some positive constant $c_{1}$ depending only on $\Omega, C,\|\mathcal{G}\|_{L^{\infty}\left(0, T ; \mathbf{W}^{\prime}\right)},\|\alpha\|_{L^{\infty}}(\mathbf{R})$, and $c_{K}$. In addition, if $\hat{\theta}$ and $\hat{\chi}_{2}$ belong to $H^{1}(0, T ; H)$ and if $\mathcal{G}$ fulfills $(1.28)$, then it follows

$$
\begin{aligned}
& \mathbf{u} \in H^{1}(0, T ; \mathbf{W}), \\
& \operatorname{div} \mathbf{u} \in H^{1}\left(0, T ; H^{2}(\Omega)\right) .
\end{aligned}
$$

Next, we aim to approximate the Problem $P$ by use of a semi-implicit time discretization scheme. Thus, letting $N$ be an arbitrary positive integer, we denote by $\tau:=T / N$ the time step of our backward finite differences scheme. Then, by easily adapting the argument of Lemma 2.1, and due to (1.29)-(1.30) (cf. (1.13) and (1.14)) and (1.28), we can introduce the vector of initial displacements $\mathbf{u}_{0} \in \mathbf{W}$ defined as the unique solution of the abstract equation (cf. (1.17))

$$
\mathcal{H} \mathbf{u}_{0}+\mathcal{B}\left(\alpha\left(\theta_{0}\right) \chi_{20}\right)=\mathcal{G}(0) .
$$


Moreover, as it is usual in time discretization procedures, we approximate the functions $\mathcal{R}=\mathcal{R}_{1}+\mathcal{R}_{2}$ and $\mathcal{G}$, specified by (1.9)-(1.10), by two vectors $\left\{\mathcal{R}^{i}\right\}$ and $\left\{\mathcal{G}^{i}\right\}$, which are constructed as follows (cf. (1.27) and (1.28)):

$$
\begin{aligned}
& \mathcal{R}^{i}=\mathcal{R}_{1}^{i}+\mathcal{R}_{2}^{i}:=\frac{1}{\tau} \int_{(i-1) \tau}^{i \tau} \mathcal{R}_{1}(\cdot, t) d t+\mathcal{R}_{2}(\cdot, i \tau) \in H+V^{\prime}, \\
& \mathcal{G}^{i}:=\mathcal{G}^{i}(\cdot, i \tau) \in \mathbf{W}^{\prime}
\end{aligned}
$$

for $i=1, \ldots, N$, and set $\mathcal{G}^{0}=\mathcal{G}(0)$ (cf. (2.6)). Then, the approximated problem $P_{\tau}$ can be formulated as in a moment. Let us note in advance that, in the approximating form, we set the discretization of the variational inclusion (1.16) in $H^{2}$ by substituting the abstract operator $\partial I_{K, V}$ by the corresponding maximal monotone graph in $H^{2}, \partial I_{K}$, provided we can prove some regularity of the solutions. For the sake of completeness, we recall that (cf. [4])

$$
\begin{aligned}
& \left(\xi_{1}, \xi_{2}\right) \in \partial I_{K}\left(\chi_{1}, \chi_{2}\right) \text { if and only if }\left(\chi_{1}, \chi_{2}\right) \in K \text { and } \\
& \sum_{i=1}^{2} \int_{\Omega} \xi_{i}\left(x_{i}-\chi_{i}\right) \leq 0 \quad \forall\left(x_{1}, x_{2}\right) \in K .
\end{aligned}
$$

As a consequence, we will be able to solve the discrete variational inclusion a.e. in $Q$ (cf. also Remark 1.1).

Problem $P_{\tau}$. Find vectors

$$
\begin{aligned}
\left(\Theta^{0}, \Theta^{1}, \ldots, \Theta^{N}\right) & \in V^{N+1} \\
\left(\mathbf{U}^{0}, \mathbf{U}^{1}, \ldots, \mathbf{U}^{N}\right) & \in \mathbf{W}^{N+1} \\
\left(\mathcal{X}_{1}^{0}, \mathcal{X}_{1}^{1}, \ldots, \mathcal{X}_{1}^{N}\right) & \in H^{2}(\Omega)^{N+1}, \\
\left(\mathcal{X}_{2}^{0}, \mathcal{X}_{2}^{1}, \ldots, \mathcal{X}_{2}^{N}\right) & \in H^{2}(\Omega)^{N+1},
\end{aligned}
$$

satisfying

$$
\Theta^{0}=\theta_{0}, \quad \mathbf{U}^{0}=\mathbf{u}_{0}, \quad \mathcal{X}_{1}^{0}=\chi_{10}, \quad \mathcal{X}_{2}^{0}=\chi_{20},
$$

and such that, by letting $Z^{i}=\operatorname{div} \mathbf{U}^{i}$, the following equations hold for $i=1, \ldots, N$ :

$$
\begin{aligned}
& F\left(\Theta^{i-1}, \mathbf{U}^{i-1}, \mathcal{X}_{1}^{i-1}, \mathcal{X}_{2}^{i-1}\right) \frac{\Theta^{i}-\Theta^{i-1}}{\tau}+A \Theta^{i} \\
& =\mathcal{R}^{i}+\Theta^{i-1} \lambda^{\prime}\left(\Theta^{i-1}\right) \frac{\mathcal{X}_{1}^{i}-\mathcal{X}_{1}^{i-1}}{\tau}+\Theta^{i-1} \alpha^{\prime}\left(\Theta^{i-1}\right) Z^{i-1} \frac{\mathcal{X}_{2}^{i}-\mathcal{X}_{2}^{i-1}}{\tau} \\
& +\Theta^{i-1} \alpha^{\prime}\left(\Theta^{i-1}\right) \mathcal{X}_{2}^{i-1} \frac{Z^{i}-Z^{i-1}}{\tau}+\sum_{j=1}^{2}\left(\frac{\mathcal{X}_{j}^{i}-\mathcal{X}_{j}^{i-1}}{\tau}\right)^{2} \text { in } V^{\prime}, \\
& \tau^{-1}\left(\begin{array}{l}
\mathcal{X}_{1}^{i}-\mathcal{X}_{1}^{i-1} \\
\mathcal{X}_{2}^{i}-\mathcal{X}_{2}^{i-1}
\end{array}\right)+\tau^{-1}\left(\begin{array}{l}
A \mathcal{X}_{1}^{i}-A \mathcal{X}_{1}^{i-1} \\
A \mathcal{X}_{2}^{i}-A \mathcal{X}_{2}^{i-1}
\end{array}\right)+\left(\begin{array}{l}
A \mathcal{X}_{1}^{i} \\
A \mathcal{X}_{2}^{i}
\end{array}\right)+\left(\begin{array}{c}
\Xi_{1}^{i} \\
\Xi_{2}^{i}
\end{array}\right) \\
& =\left(\begin{array}{c}
-\lambda\left(\Theta^{i}\right) \\
-\alpha\left(\Theta^{i}\right) Z^{i}
\end{array}\right) \text { in } H^{2} \\
& \mathcal{H} \mathbf{U}^{i}+\mathcal{B}\left(\alpha\left(\Theta^{i}\right) \mathcal{X}_{2}^{i}\right)=\mathcal{G}^{i} \text { in } \mathbf{W}^{\prime},
\end{aligned}
$$


for

$$
\left(\Xi_{1}^{i}, \Xi_{2}^{i}\right) \in \partial I_{K}\left(\mathcal{X}_{1}^{i}, \mathcal{X}_{2}^{i}\right)
$$

and $F$ being specified by (1.18).

REMARK 2.1. In view of (2.6), (2.17), (2.8), and Lemma 2.1, it is not difficult to check that for $i=0, \ldots, N$ the function $Z^{i}$ satisfies (2.3) with the same constant $c_{1}$. Thus, the positions (2.6)-(2.8) and (2.10)-(2.14) allow us to give a meaning to (2.15) and (2.16), which contain the explicit terms of the above scheme.

Let us note that the function $F$ in (1.15) represents the specific heat of the phase transition (as it is the coefficient of the time derivative of the temperature in the energy balance) and consequently it seems physically consistent to require it is positive everywhere. Thus, after observing that by (1.11), (2.3), (1.20), and (1.19), we have

$$
F\left(\theta, \mathbf{u}, \chi_{1}, \chi_{2}\right) \geq c_{s}-c_{\lambda}\left(1+c_{K}\right)-\theta_{c} c_{\alpha} c_{1} c_{K}
$$

we require that

$$
c_{2}:=c_{s}-c_{\lambda}\left(1+c_{K}\right)-\theta_{c} c_{\alpha} c_{1} c_{K}>0,
$$

which is sufficient to ensure that $F$ is positive a.e. in $Q$. Henceforth, in the following lemma we establish the existence of a solution for the approximating discrete problem $P_{\tau}$, at any step $\tau>0$.

LEMma 2.2. Under the assumptions (1.29)-(1.30), (1.19)-(1.20), (2.7)-(2.8), and (2.20), for any $\tau>0$ the problem $P_{\tau}$ admits a solution.

Proof. Owing to (2.6), (2.14), and (1.29)-(1.30), we can restrict ourselves to prove that for any fixed $\tau>0$ and for any $i \geq 1$, the system (2.15)-(2.18) admits a solution. The main idea is to proceed by induction on $i$. Indeed, we suppose to know

$$
\left(\Theta^{i-1}, \mathbf{U}^{i-1},\left(\mathcal{X}_{1}^{i-1}, \mathcal{X}_{2}^{i-1}\right)\right) \in V \times \mathbf{W} \times H^{2}(\Omega)^{2} \cap K
$$

and $Z^{i-1}$ fulfilling (2.3) (see (2.10)-(2.13)). We look for

$$
\left(\Theta^{i}, \mathbf{U}^{i},\left(\mathcal{X}_{1}^{i}, \mathcal{X}_{2}^{i}\right)\right) \in V \times \mathbf{W} \times H^{2}(\Omega)^{2} \cap K
$$

solving the resulting equations $(2.15)-(2.17)$. To this aim, we perform a fixed point argument; namely, by use of the Schauder theorem we shall prove that a suitable operator $D: H^{2} \rightarrow H^{2}$ admits at least a fixed point.

We first fix

$$
\left(\widetilde{\Theta}, \widetilde{\mathcal{X}}_{2}\right) \in H^{2} \text { with }\left|\tilde{\chi}_{2}\right| \leq c_{K}
$$

and substitute $\Theta^{i}$ and $\mathcal{X}_{2}^{i}$ in (2.17). Thus, due to (1.20), (2.8), and (2.23), we can refer to the first part of Lemma 2.1 and find a unique corresponding solution

$$
\mathbf{U}^{i}=D_{1}\left(\widetilde{\Theta}, \widetilde{\mathcal{X}}_{2}\right) \in \mathbf{W},
$$

such that $\operatorname{div} \mathbf{U}^{i}=Z^{i}$ fulfills (2.3) (for any $i$ ).

On a second step, we write $\widetilde{\Theta}$ instead of $\Theta^{i}$ in $(2.16)$ and fix $\mathbf{U}^{i}=D_{1}\left(\widetilde{\Theta}, \widetilde{\mathcal{X}}_{2}\right)$. Known results on elementary abstract equations with maximal monotone operators ensure the existence and uniqueness of a pair of functions

$$
\left(\mathcal{X}_{1}^{i}, \mathcal{X}_{2}^{i}\right)=D_{2}\left(\widetilde{\Theta}, \mathbf{U}^{i}\right) \in H^{2}(\Omega)^{2} \cap K
$$


fulfilling in $H^{2}$

$$
\begin{array}{r}
\tau^{-1}\left(\begin{array}{c}
\mathcal{X}_{1}^{i} \\
\mathcal{X}_{2}^{i}
\end{array}\right)+\left(1+\tau^{-1}\right)\left(\begin{array}{c}
A \mathcal{X}_{1}^{i} \\
A \mathcal{X}_{2}^{i}
\end{array}\right)+\left(\begin{array}{c}
\Xi_{1}^{i} \\
\Xi_{2}^{i}
\end{array}\right) \\
=\left(\begin{array}{c}
\tau^{-1}\left(\mathcal{X}_{1}^{i-1}+A \mathcal{X}_{1}^{i-1}\right)-\lambda(\widetilde{\Theta}) \\
\tau^{-1}\left(\mathcal{X}_{2}^{i-1}+A \mathcal{X}_{2}^{i-1}\right)-\alpha(\widetilde{\Theta}) Z^{i}
\end{array}\right),
\end{array}
$$

for $\left(\Xi_{1}^{i}, \Xi_{2}^{i}\right) \in \partial I_{K}\left(\mathcal{X}_{1}^{i}, \mathcal{X}_{2}^{i}\right)$. We can prove the above result owing to (2.21), (1.19)-(1.20), and (2.3). Indeed, as the right hand side, called, i.e., $\mathcal{F}$, of $(2.26)$ is known in $H^{2}$, the above relation can be equivalently rewritten as

$$
\left(\tau^{-1} I d+\mathcal{A}+\partial I_{K}\right)\left(\mathcal{X}_{1}^{i}, \mathcal{X}_{2}^{i}\right) \ni \mathcal{F}
$$

where $I d$ stands for the identity operator in $H^{2}$ and (cf. (1.6))

$$
\mathcal{A}\left(\mathcal{X}_{1}^{i}, \mathcal{X}_{2}^{i}\right)=\left(1+\tau^{-1}\right)\left(A \mathcal{X}_{1}^{i}, A \mathcal{X}_{2}^{i}\right)
$$

Thus, we can approximate the subdifferential operator in (2.27) by its Yosida regularization and solve the approximated equation owing to well known results on the sum of maximal monotone operators (cf. [4, Lemme 2.4, p. 34]). Hence, a standard a priori estimates-passage to the limit procedure leads to (2.25) solving (2.27) (cf. [6, Appendix]). On a second step we can test $(2.26)$ by $\left(\mathcal{X}_{1}^{i}, \mathcal{X}_{2}^{i}\right)$ and exploit monotonicity arguments to show that $\sum_{j=1}^{2} \int_{\Omega} \Xi_{j}^{i} \mathcal{X}_{j}^{i} \geq 0$, as $(0,0) \in \partial I_{K}(0,0)$. Thus, by virtue of the Young inequality, and owing to $(1.20),(1.19)$, and $(2.3)$, we can write

$$
\begin{aligned}
& \frac{1}{\tau} \sum_{j=1}^{2}\left\|\mathcal{X}_{j}^{i}\right\|_{H}^{2}+\left(1+\frac{1}{\tau}\right) \int_{\Omega}\left|\nabla \mathcal{X}_{j}^{i}\right|^{2} \\
& \quad \leq \frac{1}{2 \tau} \sum_{j=1}^{2}\left\|\mathcal{X}_{j}^{i}\right\|_{I^{2}}^{2}+\tau|\Omega|\left(\|\lambda\|_{L^{\infty}(\mathbf{R})}^{2}+\|\alpha\|_{L^{\infty}(\mathbf{R})}^{2} c_{1}^{2}\right)+c \sum_{j=1}^{2}\left\|\mathcal{X}_{j}^{i-1}\right\|_{V}^{2},
\end{aligned}
$$

where $|\Omega|$ stands for the Lebesgue measure of $\Omega$, and finally deduce (cf. (2.21))

$$
\sum_{j=1}^{2}\left\|\mathcal{X}_{j}^{i}\right\|_{V}^{2} \leq c
$$

for a suitable positive constant $c$ depending on the explicit terms in $(2.26)$, on $\tau, c_{1}$,

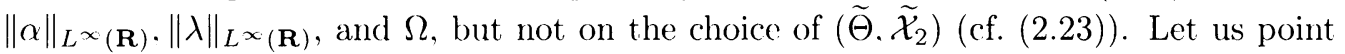
out in addition that, as $\left(\mathcal{X}_{1}^{i}, \mathcal{X}_{2}^{i}\right)$ solve $(2.27),\left(\mathcal{X}_{1}^{i}, \mathcal{X}_{2}^{i}\right) \in K$ and consequently fulfil (1.11). We also note that here and in the sequel the same notation $c$ stands for possibly different constants, depending on the data of the problem and the parameters which from row to row are considered as constants.

Finally. we take in $(2.15)$ the functions $\left(\mathcal{X}_{1}^{i}, \mathcal{X}_{2}^{i}\right)=D_{2}\left(\widetilde{\Theta}, D_{1}\left(\tilde{\Theta}, \tilde{\mathcal{X}}_{2}\right)\right)$ and $\mathbf{U}^{i}=$ $D_{1}\left(\tilde{\Theta}, \widetilde{\mathcal{X}}_{2}\right)$. Hence, by exploiting once more the Lax-Milgram theorem, we can find a unique function

$$
\Theta^{i}=D_{3}\left(\mathbf{U}^{i}, \mathcal{X}_{1}^{i}, \mathcal{X}_{2}^{i}\right) \in V
$$


solving in $V^{\prime}$ the resulting equation

$$
\begin{aligned}
& \tau^{-1} F\left(\Theta^{i-1}, \mathbf{U}^{i-1}, \mathcal{X}_{1}^{i-1}, \mathcal{X}_{2}^{i-1}\right) \Theta^{i}+A \Theta^{i}=\tau^{-1} F\left(\Theta^{i-1}, \mathbf{U}^{i-1}, \mathcal{X}_{1}^{i-1}, \mathcal{X}_{2}^{i-1}\right) \Theta^{i-1} \\
& +\mathcal{R}^{i}+\Theta^{i-1} \lambda^{\prime}\left(\Theta^{i-1}\right) \frac{\mathcal{X}_{1}^{i}-\mathcal{X}_{1}^{i-1}}{\tau}+\Theta^{i-1} \alpha^{\prime}\left(\Theta^{i-1}\right) Z^{i-1} \frac{\mathcal{X}_{2}^{i}-\mathcal{X}_{2}^{i-1}}{\tau} \\
& +\Theta^{i-1} \alpha^{\prime}\left(\Theta^{i-1}\right) \mathcal{X}_{2}^{i-1} \frac{Z^{i}-Z^{i-1}}{\tau}+\sum_{j=1}^{2}\left(\frac{\mathcal{X}_{j}^{i}-\mathcal{X}_{j}^{i-1}}{\tau}\right)^{2},
\end{aligned}
$$

where the right hand side is known in $H+V^{\prime}$. More precisely, notice that the following inequalities are straightforward (cf. (2.20), (1.19)-(1.20), (1.11), and (2.3)):

$$
0<c_{2} \leq F\left(\Theta^{i-1}, \mathbf{U}^{i-1}, \mathcal{X}_{1}^{i-1}, \mathcal{X}_{2}^{i-1}\right) \leq c_{s}+c_{\lambda}\left(1+c_{K}\right)+\theta_{c} c_{\alpha} c_{K} c_{1} .
$$

Thus, it is clear that the left hand side of (2.31) is associated to a continuous bilinear form, which turns out to be $V$-coercive, while the right hand side represents a continuous linear operator on $V$. Thus, Lax-Milgram theorem is applied to get the required existence and uniqueness result (2.30). Finally, we can prove a uniform bound for $\Theta^{i}$, independent of the choice of $\widetilde{\Theta}$ and $\widetilde{\mathcal{X}}_{2}$. Indeed, if we test $(2.31)$ by $\Theta^{i}$, owing to (1.19)-(1.20), (2.3), (1.11), and (2.32), thanks to the Young inequality we can easily get

$$
\left\|\Theta^{i}\right\|_{V} \leq c
$$

for a constant $c$ depending on the explicit terms of $(2.31), \tau, c_{1}, c_{2}, c_{K}, \Omega, c_{\alpha}, \theta_{c}, c_{\lambda}, \tilde{c}_{\lambda}$, and $\left\|\mathcal{R}^{i}\right\|_{H+V^{\prime}}$.

By the above arguments, it turns out that the operator $D: H^{2} \rightarrow H^{2}$, specified by

$$
D\left(\widetilde{\Theta}, \tilde{\chi}_{2}\right):=\left(D_{3}\left(D_{1}\left(\widetilde{\Theta}, \tilde{\mathcal{X}}_{2}\right), \mathcal{X}_{1}^{i}, \mathcal{X}_{2}^{i}\right), \mathcal{X}_{2}^{i}\right),\left(\mathcal{X}_{1}^{i}, \mathcal{X}_{2}^{i}\right)=D_{2}\left(\widetilde{\Theta}, D_{1}\left(\widetilde{\Theta}, \tilde{\mathcal{X}}_{2}\right)\right) \text {. }
$$

In particular, we observe that (2.29) and (2.33) imply that $D$ is a compact operator in $H^{2}$. Thus, in order to achieve the proof by Schauder's fixed point theorem, it remains to show that $D$ is continuous with respect to the natural topology of $H^{2}$. This property can be verified by exploiting contracting arguments and showing that the operators $D_{1}, D_{2}$, and $D_{3}$ are Lipschitz continuous.

To this aim, we first write $(2.17)$ for $\left(\widetilde{\Theta}_{1}, \widetilde{\mathcal{X}}_{21}\right)$ and $\left(\widetilde{\Theta}_{2}, \widetilde{\mathcal{X}}_{22}\right)$ fulfilling $(2.23)$, denote by $\mathbf{U}_{1}^{i}$ and $\mathbf{U}_{2}^{i}$ the corresponding solutions, take the difference of the two resulting equations

$$
\mathcal{H}\left(\mathbf{U}_{1}^{i}-\mathbf{U}_{2}^{i}\right)+\mathcal{B}\left(\alpha\left(\widetilde{\Theta}_{1}\right), \tilde{\mathcal{X}}_{21}-\alpha\left(\widetilde{\Theta}_{2}\right) \tilde{\mathcal{X}}_{22}\right)=0
$$

and test it by $\mathbf{U}_{1}^{i}-\mathbf{U}_{2}^{i}$. With the help of (1.4), (1.20), and (1.11), we can infer that

$$
\begin{aligned}
& C\left\|\mathbf{U}_{1}^{i}-\mathbf{U}_{2}^{i}\right\|_{\mathbf{W}}^{2} \leq \int_{\Omega}\left(\left|\alpha\left(\widetilde{\Theta}_{1}\right)-\alpha\left(\widetilde{\Theta}_{2}\right)\right|\left|\widetilde{\mathcal{X}}_{21}\right|+\left|\widetilde{\mathcal{X}}_{21}-\widetilde{\mathcal{X}}_{22}\right|\left|\alpha\left(\widetilde{\Theta}_{2}\right)\right|\right)\left|Z_{1}^{i}-Z_{2}^{i}\right| \\
& \leq \theta_{c} c_{\alpha} c_{K}\left\|\widetilde{\Theta}_{1}-\widetilde{\Theta}_{2}\right\|_{H}\left\|Z_{1}^{i}-Z_{2}^{i}\right\|_{H}+\|\alpha\|_{L^{\infty}(\mathbf{R})}\left\|\widetilde{\mathcal{X}}_{21}-\widetilde{\mathcal{X}}_{22}\right\|_{H}\left\|Z_{1}^{i}-Z_{2}^{i}\right\|_{H},
\end{aligned}
$$

and then, by recalling (1.2), we apply the Young inequality and get

$$
\left\|\mathbf{U}_{1}^{i}-\mathbf{U}_{2}^{i}\right\|_{\mathbf{W}}^{2} \leq c\left(\left\|\widetilde{\Theta}_{1}-\widetilde{\Theta}_{2}\right\|_{H}^{2}+\left\|\tilde{\mathcal{X}}_{21}-\tilde{\mathcal{X}}_{22}\right\|_{H}^{2}\right)
$$

for a constant $c$ which depends on $C,\|\alpha\|_{L^{\infty}(\mathbf{R})}, \theta_{c}, c_{\alpha}$, and $c_{K}$. 
Then, we write $(2.26)$ for $\left(\widetilde{\Theta}_{1}, \mathbf{U}_{1}^{i}\right)$ and $\left(\widetilde{\Theta}_{2}, \mathbf{U}_{2}^{i}\right)$, take the difference and, after observing that the explicit terms cancel, we write

$$
\begin{gathered}
\tau^{-1}\left(\begin{array}{l}
\mathcal{X}_{11}^{i}-\mathcal{X}_{12}^{i} \\
\mathcal{X}_{21}^{i}-\mathcal{X}_{22}^{i}
\end{array}\right)+\left(\tau^{-1}+1\right)\left(\begin{array}{l}
A\left(\mathcal{X}_{11}^{i}-\mathcal{X}_{12}^{i}\right) \\
A\left(\mathcal{X}_{21}^{i}-\mathcal{X}_{22}^{i}\right)
\end{array}\right) \\
+\left(\begin{array}{l}
\Xi_{11}^{i}-\Xi_{12}^{i} \\
\Xi_{21}^{i}-\Xi_{22}^{i}
\end{array}\right)=\left(\begin{array}{c}
-\left(\lambda\left(\widetilde{\Theta}_{1}\right)-\lambda\left(\widetilde{\Theta}_{2}\right)\right) \\
-\left(\alpha\left(\widetilde{\Theta}_{1}\right) Z_{1}^{i}-\alpha\left(\widetilde{\Theta}_{2}\right) Z_{2}^{i}\right)
\end{array}\right) .
\end{gathered}
$$

Thus if we test $(2.38)$ by the difference of the corresponding solutions $\left(\mathcal{X}_{11}^{i}-\mathcal{X}_{12}^{i}, \mathcal{X}_{21}^{i}-\right.$ $\mathcal{X}_{22}^{i}$ ), the monotonicity of the subdifferential operator ensures

$$
\sum_{j=1}^{2} \int_{\Omega}\left(\Xi_{j 1}^{i}-\Xi_{j 2}^{i}\right)\left(\mathcal{X}_{j 1}^{i}-\mathcal{X}_{j 2}^{i}\right) \geq 0
$$

since $\left(\Xi_{1 j}^{i}, \Xi_{2 j}^{i}\right) \in \partial I_{K}\left(\mathcal{X}_{1 j}, \mathcal{X}_{2 j}\right)$. By similarly proceeding as above, and recalling (1.20), $(1.19)$, and (2.3), we finally get

$$
\sum_{j=1}^{2}\left\|\mathcal{X}_{j 1}^{i}-\mathcal{X}_{j 2}^{i}\right\|_{V}^{2} \leq c\left(\left\|\widetilde{\Theta}_{1}-\widetilde{\Theta}_{2}\right\|_{H}^{2}+\left\|\mathbf{U}_{1}^{i}-\mathbf{U}_{2}^{i}\right\|_{\mathbf{W}}^{2}\right)
$$

for $c$ depending on $\tau, c_{1},\|\alpha\|_{W^{1, \infty}(\mathbf{R})}$, and $\|\lambda\|_{W^{1, \infty}(\mathbf{R})}$ (i.e. on $\theta_{c}, c_{\alpha}$, and $\tilde{c}_{\lambda}$ ).

Finally, we write $(2.31)$ for $\left(\mathbf{U}_{1}^{i}, \mathcal{X}_{11}^{i}, \mathcal{X}_{21}^{i}\right)$ and $\left(\mathbf{U}_{2}^{i}, \mathcal{X}_{12}^{i}, \mathcal{X}_{22}^{i}\right)$, take the difference so that the explicit terms cancel, and write the equality

$$
\begin{aligned}
\tau^{-1} F & \left(\Theta^{i-1}, \mathbf{U}^{i-1}, \mathcal{X}_{1}^{i-1}, \mathcal{X}_{2}^{i-1}\right)\left(\Theta_{1}^{i}-\Theta_{2}^{i}\right)+A\left(\Theta_{1}^{i}-\Theta_{2}^{i}\right) \\
= & \Theta^{i-1} \lambda^{\prime}\left(\Theta^{i-1}\right) \frac{\mathcal{X}_{11}^{i}-\mathcal{X}_{12}^{i}}{\tau}+\Theta^{i-1} \alpha^{\prime}\left(\Theta^{i-1}\right) Z^{i-1} \frac{\mathcal{X}_{21}^{i}-\mathcal{X}_{22}^{i}}{\tau} \\
& +\Theta^{i-1} \alpha^{\prime}\left(\Theta^{i-1}\right) \mathcal{X}_{2}^{i-1} \frac{Z_{1}^{i}-Z_{2}^{i}}{\tau} \\
& +\sum_{j=1}^{2}\left(\frac{\mathcal{X}_{j 1}^{i}-\mathcal{X}_{j 2}^{i}}{\tau}\right)\left(\frac{\mathcal{X}_{j 1}^{i}+\mathcal{X}_{j 2}^{i}-2 \mathcal{X}_{j}^{i-1}}{\tau}\right) .
\end{aligned}
$$

Hence, if we test $(2.40)$ by $\Theta_{1}^{i}-\Theta_{2}^{i}$, due to $(2.20),(1.20),(1.19),(2.3),(1.2)$, and (1.11), by applying the Hölder inequality, it is now a standard matter to infer that

$$
\left\|\Theta_{1}^{i}-\Theta_{2}^{i}\right\|_{V}^{2} \leq c\left(\left\|\mathbf{U}_{1}^{i}-\mathbf{U}_{2}^{i}\right\|_{\mathbf{W}}^{2}+\sum_{j=1}^{2}\left\|\mathcal{X}_{j 1}^{i}-\mathcal{X}_{j 2}^{i}\right\|_{H}^{2}\right)
$$

for $c$ depending on $\tau, c_{1}, c_{2}, c_{K}, c_{\alpha}, \theta_{c}$, and $\tilde{c}_{\lambda}$.

Now, we can combine (2.37), (2.39), and (2.41) and claim

$$
\left\|\Theta_{1}^{i}-\Theta_{2}^{i}\right\|_{H}^{2}+\left\|\mathcal{X}_{21}^{i}-\mathcal{X}_{22}^{i}\right\|_{H}^{2} \leq c\left(\left\|\widetilde{\Theta}_{1}-\widetilde{\Theta}_{2}\right\|_{H}^{2}+\left\|\widetilde{\mathcal{X}}_{21}-\widetilde{\mathcal{X}}_{22}\right\|_{H}^{2}\right),
$$

which ensures that the operator $D$ introduced in (2.34) is Lipschitz continuous in $H^{2}$. Thus, the hypotheses of the Schauder theorem are satisfied by $D$ and consequently we can deduce that it admits a fixed point $\left(\Theta^{i}, \mathcal{X}_{2}^{i}\right)$. This concludes our proof of Lemma 2.2 , since for any $i$, and any fixed $\tau>0$, it is now clear that the corresponding quadruple $\left(\Theta^{i}, \mathbf{U}^{i}, \mathcal{X}_{1}^{i}, \mathcal{X}_{2}^{i}\right)$ turns out to solve the system (2.15)-(2.17). 
3. A priori estimates. Now, we aim to establish some a priori estimates independent of $\tau$, holding at least for $\tau \in(0, \hat{\tau})$ with $\hat{\tau}$ sufficiently small, on the time-discrete solutions. Thus, we refer to the existence result which has been previously stated by Lemma 2.2. Moreover, we require that the following inequality holds:

$$
\left(\theta_{c} c_{\alpha} c_{K}\left(\theta_{c}+1\right)\right)^{2} \leq c_{2}\left(\lambda_{L}+2 \mu_{L} / 3\right),
$$

and observe that (2.20) and (3.1) make eventually precise (1.22).

For the sake of clarity, we introduce some useful notations. Let $\left\{a^{i}\right\}, i=0,1, \ldots, N$, be a vector. Then, we denote by $a_{\tau}$ and $\tilde{a}_{\tau}$ two functions defined on $(-\infty, T]$ and $[0, T]$ which interpolate the values of the vector piecewise linearly and backward constantly, respectively. That is

$$
\begin{aligned}
& a_{\tau}(t):=a^{0} \text { if } t \leq 0 \\
& a_{\tau}(t):=a^{i} \text { if } t \in((i-1) \tau, i \tau], \\
& \tilde{a}_{\tau}(t):=a^{i}+\frac{a^{i}-a^{i-1}}{\tau}(t-i \tau) \text { if } t \in[(i-1) \tau, i \tau],
\end{aligned}
$$

for $i=1, \ldots, N$. In addition, let $\mathcal{I}_{\tau}$ denote the one-step backward translation operator of the scheme, namely

$$
\begin{aligned}
& \mathcal{I}_{\tau} f(x, t):=f(x, t-\tau) \text { for a.e. }(x, t) \in \Omega \times(0, T), \\
& \forall f \in L^{1}(\Omega \times(-\tau, T)) .
\end{aligned}
$$

Hence, we can equivalently write Eqs. (2.15) and (2.17), respectively, as follows:

$$
\begin{aligned}
\mathcal{I}_{\tau}( & \left.F\left(\theta_{\tau}, \mathbf{u}_{\tau}, \chi_{1 \tau}, \chi_{2 \tau}\right)\right) \partial_{t} \tilde{\theta}_{\tau}+A \theta_{\tau}=\mathcal{R}_{\tau}+\mathcal{I}_{\tau}\left(\theta_{\tau} \lambda^{\prime}\left(\theta_{\tau}\right)\right) \partial_{t} \tilde{\chi}_{1 \tau} \\
& +\mathcal{I}_{\tau}\left(\theta_{\tau} \alpha^{\prime}\left(\theta_{\tau}\right) z_{\tau}\right) \partial_{t} \tilde{\chi}_{2 \tau}+\mathcal{I}_{\tau}\left(\theta_{\tau} \alpha^{\prime}\left(\theta_{\tau}\right) \chi_{2 \tau}\right) \partial_{t} \tilde{z}_{\tau} \\
& +\sum_{j=1}^{2}\left(\partial_{t} \tilde{\chi}_{j \tau}\right)^{2} \text { in } V^{\prime}
\end{aligned}
$$

and

$$
\mathcal{H} \mathbf{u}_{\tau}+\mathcal{B}\left(\alpha\left(\theta_{\tau}\right) \chi_{2 \tau}\right)=\mathcal{G}_{\tau} \text { in } \mathbf{W}^{\prime}
$$

a.e. in $(0, T)$. As to the inclusion (2.16), we could write it in $H^{2}$ in terms of the above introduced piecewise linear and constant functions. Nonetheless, in order to perform a passage to the limit procedure as $\tau \searrow 0$, we have to set this inclusion in the abstract framework of the $\left(V^{\prime}\right)^{2}-V^{2}$ duality. Thus, instead of $\partial I_{K}$, we write the corresponding abstract operator $\partial I_{K, V}$ and we recall that it is the subdifferential from $(V)^{2}$ into $\left(V^{\prime}\right)^{2}$ of the indicator function $I_{K \cap V^{2}}$ of the convex $K \cap V^{2}$ (cf. (1.12)). Obviously, by the regularity of the vectors $\left\{\Theta^{i}\right\},\left\{\mathcal{X}_{1}^{i}\right\},\left\{\mathcal{X}_{2}^{i}\right\}$, and $\left\{\mathbf{U}^{i}\right\}$ (cf. (2.10)-(2.13) and (2.18)), the existence result we have proved in the previous section can be obviously extended to the abstract framework of $\left(V^{\prime}\right)^{2}$ (cf. Remark 1.1); namely, we have

$$
\begin{gathered}
\left(\begin{array}{c}
\partial_{t} \tilde{\chi}_{1 \tau} \\
\partial_{t} \tilde{\chi}_{2 \tau}
\end{array}\right)+\left(\begin{array}{c}
A \partial_{t} \tilde{\chi}_{1 \tau} \\
A \partial_{t} \tilde{\chi}_{2 \tau}
\end{array}\right)+\left(\begin{array}{c}
A \chi_{1 \tau} \\
A \chi_{2 \tau}
\end{array}\right)+\left(\begin{array}{l}
\xi_{1 \tau} \\
\xi_{2 \tau}
\end{array}\right) \\
=\left(\begin{array}{c}
-\lambda\left(\theta_{\tau}\right) \\
-\alpha\left(\theta_{\tau}\right) z_{\tau}
\end{array}\right) \text { in }\left(V^{\prime}\right)^{2}
\end{gathered}
$$


a.e. in $(0, T)$, for

$$
\left(\begin{array}{l}
\xi_{1 \tau} \\
\xi_{2 \tau}
\end{array}\right) \in \partial I_{K, V}\left(\chi_{1 \tau}, \chi_{2 \tau}\right) \text { in }\left(V^{\prime}\right)^{2} .
$$

In addition, let us observe that by construction (cf. (3.3)), $\tilde{\theta}_{\tau}, \tilde{\chi}_{1 \tau}$, and $\tilde{\chi}_{2 \tau}$ satisfy the natural Cauchy conditions (cf. (1.29) (1.30) and (2.14))

$$
\begin{aligned}
& \tilde{\theta}_{\tau}(0)=\theta_{0}, \\
& \left(\tilde{\chi}_{1 \tau}(0), \tilde{\chi}_{2 \tau}(0)\right)=\left(\chi_{10}, \chi_{20}\right),
\end{aligned}
$$

while $z_{\tau}$ and $\tilde{z}_{\tau}$ fulfil (2.3) independently of $\tau$.

Hence, we are going to prove some estimates, holding at least for $\tau$ sufficiently small, but not depending on $\tau$, on the approximating functions solving (3.5)-(3.7). Indeed, our aim is passing to the limit in the above system as $\tau \searrow 0$, by compactness or direct proof, to get (1.15)-(1.17) solved in a suitable sense.

Nonetheless, in order to prove these uniform bounds, we need to impose another restriction on the data in the same spirit of (2.20), i.e., we have to assume that (3.1) holds. In addition, let us recall the trivial equality

$$
2 a(a-b)=a^{2}+(a-b)^{2}-b^{2}, \quad \forall a, b \in \mathbf{R},
$$

which will be applied in the following estimates on the discrete solutions.

First a priori estimate. We first test $(2.16)$ by $\left(\mathcal{X}_{1}^{i}-\mathcal{X}_{1}^{i-1}, \mathcal{X}_{2}^{i}-\mathcal{X}_{2}^{i-1}\right)$. Let us observe that, by definition of the subdifferential, (2.18) yields (cf. (2.9))

$$
\sum_{j=1}^{2} \int_{\Omega} \Xi_{j}^{i}\left(\mathcal{X}_{j}^{i}-\mathcal{X}_{j}^{i-1}\right) \geq 0
$$

as $\left(\mathcal{X}_{1}^{i-1}, \mathcal{X}_{2}^{i-1}\right) \in K$ (cf. (2.21)). Hence, exploiting the relation (3.11) and Hölder's inequality, by use of (1.20), (1.19), and (2.3), we can infer

$$
\begin{aligned}
\sum_{j=1}^{2} \tau & \left(\left\|\frac{\mathcal{X}_{j}^{i}-\mathcal{X}_{j}^{i-1}}{\tau}\right\|_{H}^{2}+\int_{\Omega}\left|\frac{\nabla \mathcal{X}_{j}^{i}-\nabla \mathcal{X}_{j}^{i-1}}{\tau}\right|^{2}\right) \\
& +\frac{1}{2} \sum_{j=1}^{2} \int_{\Omega}\left(\left|\nabla \mathcal{X}_{j}^{i}\right|^{2}+\left|\nabla\left(\mathcal{X}_{j}^{i}-\mathcal{X}_{j}^{i-1}\right)\right|^{2}-\left|\nabla \mathcal{X}_{j}^{i-1}\right|^{2}\right) \\
\leq & \tau \int_{\Omega}\left|\lambda\left(\Theta^{i}\right)\right|\left|\frac{\mathcal{X}_{1}^{i}-\mathcal{X}_{1}^{i-1}}{\tau}\right|+\tau \int_{\Omega}\left|\alpha\left(\Theta^{i}\right) Z^{i}\right|\left|\frac{\mathcal{X}_{2}^{i}-\mathcal{X}_{2}^{i-1}}{\tau}\right| \\
\leq & \frac{\tau}{2}\left(\|\lambda\|_{L^{\infty}(\mathbf{R})}^{2}+\|\alpha\|_{L^{\infty}(\mathbf{R})}^{2} c_{1}^{2}\right)|\Omega|+\frac{\tau}{2} \sum_{j=1}^{2}\left\|\frac{\mathcal{X}_{j}^{i}-\mathcal{X}_{j}^{i-1}}{\tau}\right\|_{H}^{2} .
\end{aligned}
$$


Next, by summing up (3.13) for $i=1, \ldots, m$, with $m \leq N=T / \tau$, it is easy to recover (cf. (1.30) and (2.14))

$$
\begin{gathered}
\sum_{i=1}^{m} \sum_{j=1}^{2} \frac{\tau}{2}\left\|\frac{\mathcal{X}_{j}^{i}-\mathcal{X}_{j}^{i-1}}{\tau}\right\|_{V}^{2}+\frac{1}{2} \sum_{j=1}^{2} \int_{\Omega}\left|\nabla \mathcal{X}_{j}^{m}\right|^{2} \\
\leq c+\frac{1}{2} \sum_{j=1}^{2} \int_{\Omega}\left|\nabla \mathcal{X}_{j}^{0}\right|^{2} \leq c .
\end{gathered}
$$

Let us note that we can improve the regularity of the estimate (3.14). Indeed, we can test (2.16) by $\tau\left(A \mathcal{X}_{1}^{i}, A \mathcal{X}_{2}^{i}\right)$ (cf. (2.12)-(2.13) and (1.6)). By formal arguments, it is a standard matter to verify that the monotonicity of the subdifferential operator leads to (cf. [6, Appendix])

$$
\tau \sum_{i=1}^{2} \int_{\Omega} \Xi_{j}^{i} A \mathcal{X}_{j}^{i} \geq 0
$$

Hence, by exploiting once more (3.11) and Hölder's inequality, by similarly proceeding as for (3.13), we owe to (1.20), (1.19), and (2.3) and write

$$
\begin{aligned}
\sum_{j=1}^{2} \frac{1}{2} \int_{\Omega}\left|\nabla \mathcal{X}_{j}^{i}\right|^{2}-\left|\nabla \mathcal{X}_{j}^{i-1}\right|^{2}+\left|\nabla\left(\mathcal{X}_{j}^{i}-\mathcal{X}_{j}^{i-1}\right)\right|^{2} \\
\quad+\sum_{j=1}^{2} \frac{1}{2}\left(\left\|A \mathcal{X}_{j}^{i}\right\|_{H}^{2}-\left\|A \mathcal{X}_{j}^{i-1}\right\|_{H}^{2}+\left\|A\left(\mathcal{X}_{j}^{i}-\mathcal{X}_{j}^{i-1}\right)\right\|_{H}^{2}\right) \\
\quad+\tau \sum_{j=1}^{2}\left\|A \mathcal{X}_{j}^{i}\right\|_{H}^{2} \leq \frac{1}{2} \tau \sum_{j=1}^{2}\left\|A \mathcal{X}_{j}^{i}\right\|_{H}^{2}+c
\end{aligned}
$$

Thus, by summing up for $i=1, \ldots, m$, we get (cf. (1.30))

$$
\begin{gathered}
\frac{1}{2} \sum_{j=1}^{2}\left\|A \mathcal{X}_{j}^{m}\right\|_{H}^{2}+\frac{\tau}{2} \sum_{i=1}^{m} \sum_{j=1}^{2}\left\|A \mathcal{X}_{j}^{i}\right\|_{H}^{2}+\frac{1}{2} \sum_{i=1}^{m} \int_{\Omega}\left|\nabla \mathcal{X}_{j}^{m}\right|^{2} \\
\leq c+\frac{1}{2} \sum_{j=1}^{2} \int_{\Omega}\left|\nabla \mathcal{X}_{j}^{0}\right|^{2}+\frac{1}{2} \sum_{j=1}^{2}\left\|A \mathcal{X}_{j}^{0}\right\|_{H}^{2} \leq c
\end{gathered}
$$

for any $m \leq N$.

SECOND AND THIRD A PRIORI ESTIMATES. We test $(2.15)$ by $\left(\Theta^{i}-\Theta^{i-1}\right)$. In view of the equality (3.11) and by recalling (2.7), (1.19)-(1.20), (2.20), (1.11), and (2.3), the Hölder 
inequality can be applied to infer that

$$
\begin{aligned}
c_{2} \tau & \left\|\frac{\Theta^{i}-\Theta^{i-1}}{\tau}\right\|_{H}^{2}+\frac{1}{2} \int_{\Omega}\left(\left|\nabla \Theta^{i}\right|^{2}+\left|\nabla\left(\Theta^{i}-\Theta^{i-1}\right)\right|^{2}-\left|\nabla \Theta^{i-1}\right|^{2}\right) \\
\leq & \tau\left(\tilde{c}_{\lambda}\left\|\frac{\mathcal{X}_{1}^{i}-\mathcal{X}_{1}^{i-1}}{\tau}\right\|_{H}+\theta_{c}^{2} c_{\alpha} c_{1}\left\|\frac{\mathcal{X}_{2}^{i}-\mathcal{X}_{2}^{i-1}}{\tau}\right\|_{H}\right)\left\|\frac{\Theta^{i}-\Theta^{i-1}}{\tau}\right\|_{H} \\
& +\tau \theta_{c}^{2} c_{K} c_{\alpha}\left\|\frac{Z^{i}-Z^{i-1}}{\tau}\right\|_{H}\left\|\frac{\Theta^{i}-\Theta^{i-1}}{\tau}\right\|_{H}+\tau\left\|\mathcal{R}_{1}^{i}\right\|_{H}\left\|\frac{\Theta^{i}-\Theta^{i-1}}{\tau}\right\|_{H} \\
& +\tau \sum_{j=1}^{2} \int_{\Omega}\left|\frac{\mathcal{X}_{j}^{i}-\mathcal{X}_{j}^{i-1}}{\tau}\right|^{2}\left|\frac{\Theta^{i}-\Theta^{i-1}}{\tau}\right|+\left\langle\mathcal{R}_{2}^{i}, \Theta^{i}-\Theta^{i-1}\right\rangle .
\end{aligned}
$$

On a second step, we write (2.17) for $i$ and $i=1$, take the difference, and test it by $\frac{\mathbf{U}^{i}-\mathbf{U}^{i-1}}{\tau}$. Owing to (1.4), (1.5), (1.11), (1.20), and (2.8), by use of the Hölder inequality, we can find a positive constant $c$, depending in particular on $C, c_{K}$, and $\|\alpha\|_{L^{\infty}(\mathbf{R})}$, such that the following estimate holds:

$$
\begin{gathered}
\frac{C}{4} \tau\left\|\frac{\mathbf{U}^{i}-\mathbf{U}^{i-1}}{\tau}\right\|_{\mathbf{W}}^{2}+\frac{\lambda_{L}+2 \mu_{L} / 3}{2} \tau\left\|\frac{Z^{i}-Z^{i-1}}{\tau}\right\|_{H}^{2} \\
\leq c \tau\left(\left\|\frac{\mathcal{X}_{2}^{i}-\mathcal{X}_{2}^{i-1}}{\tau}\right\|_{H}^{2}+\left\|\frac{\mathcal{G}^{i}-\mathcal{G}^{i-1}}{\tau}\right\|_{\mathbf{W}^{\prime}}^{2}\right) \\
\quad+\tau \theta_{c} c_{\alpha} c_{K}\left\|\frac{Z^{i}-Z^{i-1}}{\tau}\right\|_{H}\left\|\frac{\Theta^{i}-\Theta^{i-1}}{\tau}\right\|_{H} .
\end{gathered}
$$

Indeed, (1.4) and (1.5) imply

$$
2 a\left(\mathbf{U}^{i}-\mathbf{U}^{i-1}, \mathbf{U}^{i}-\mathbf{U}^{i-1}\right) \geq C\left\|\mathbf{U}^{i}-\mathbf{U}^{i-1}\right\|_{\mathbf{W}}^{2}+\left(\lambda_{L}+2 \mu_{L} / 3\right)\left\|Z^{i}-Z^{i-1}\right\|_{H}^{2},
$$

while the Lipschitz continuity of $\alpha$ (cf. (1.20)) and the definition of W-norm (cf. (1.2)) enable us to infer that (cf. (1.8))

$$
\begin{aligned}
\int_{\Omega} \mid \alpha & \left(\Theta^{i}\right) \mathcal{X}_{2}^{i}-\alpha\left(\Theta^{i-1}\right) \chi_{2}^{i-1}|| Z^{i}-Z^{i-1} \mid \\
\leq & \int_{\Omega}\left(\left|\alpha\left(\Theta^{i}\right)-\alpha\left(\Theta^{i-1}\right)\right|\left|\chi_{2}^{i-1}\right|+\left|\alpha\left(\Theta^{i}\right)\right|\left|\mathcal{X}_{2}^{i}-\mathcal{X}_{2}^{i-1}\right|\right)\left|Z^{i}-Z^{i-1}\right| \\
\leq & c_{K} \theta_{c} c_{\alpha}\left\|\Theta^{i}-\Theta^{i-1}\right\|_{H}\left\|Z^{i}-Z^{i-1}\right\|_{H} \\
& +\sqrt{3}\|\alpha\|_{L^{\infty}(\mathbf{R})}\left\|\mathcal{X}_{2}^{i}-\mathcal{X}_{2}^{i-1}\right\|_{H}\left\|\mathbf{U}^{i}-\mathbf{U}^{i-1}\right\|_{\mathbf{w}}
\end{aligned}
$$

Finally, we can easily get

$$
\left|\tau^{-1}\left\langle\mathcal{G}^{i}-\mathcal{G}^{i-1}, \mathbf{U}^{i}-\mathbf{U}^{i-1}\right\rangle\right| \leq \tau\left\|\frac{\mathcal{G}^{i}-\mathcal{G}^{i-1}}{\tau}\right\|_{\mathbf{W}^{\prime}}\left\|\frac{\mathbf{U}^{i}-\mathbf{U}^{i-1}}{\tau}\right\|_{\mathbf{W}} .
$$

Thus, by use of Young's inequality, due to (3.19)-(3.21), we can finally deduce (3.18). 
Now, we can combine the estimates (3.17) and (3.18) and sum them. By use of the extended Hölder inequality, we easily obtain

$$
\begin{aligned}
\frac{3 c_{2}}{4} \tau \| & \frac{\Theta^{i}-\Theta^{i-1}}{\tau} \|_{H}^{2}+\frac{1}{2} \int_{\Omega}\left(\left|\nabla \Theta^{i}\right|^{2}-\left|\nabla \Theta^{i-1}\right|^{2}+\left|\nabla\left(\Theta^{i}-\Theta^{i-1}\right)\right|^{2}\right) \\
& +\tau \frac{C}{4}\left\|\frac{\mathbf{U}^{i}-\mathbf{U}^{i-1}}{\tau}\right\|_{\mathbf{W}}^{2}+\tau \frac{\lambda_{L}+2 \mu_{L} / 3}{2}\left\|\frac{Z^{i}-Z^{i-1}}{\tau}\right\|_{H}^{2} \\
\leq & c \tau\left(\sum_{j=1}^{2}\left\|\frac{\mathcal{X}_{j}^{i}-\mathcal{X}_{j}^{i-1}}{\tau}\right\|_{H}^{2}+\sum_{j=1}^{2}\left\|\frac{\mathcal{X}_{j}^{i}-\mathcal{X}_{j}^{i-1}}{\tau}\right\|_{L^{4}(\Omega)}^{4}\right) \\
& +\tau \theta_{c} c_{\alpha} c_{K}\left(1+\theta_{c}\right)\left\|\frac{Z^{i}-Z^{i-1}}{\tau}\right\|_{H}\left\|\frac{\Theta^{i}-\Theta^{i-1}}{\tau}\right\|_{H}+\left\langle\mathcal{R}_{2}^{i}, \Theta^{i}-\Theta^{i-1}\right\rangle \\
& +c \tau\left(\left\|\frac{\mathcal{G}^{i}-\mathcal{G}^{i-1}}{\tau}\right\|_{\mathbf{W}^{\prime}}^{2}+\left\|\mathcal{R}_{1}^{i}\right\|_{H}^{2}\right) .
\end{aligned}
$$

Hence, due to (3.1), we claim

$$
\begin{gathered}
\tau \theta_{c} c_{\alpha} c_{K}\left(1+\theta_{c}\right)\left\|\frac{Z^{i}-Z^{i-1}}{\tau}\right\|_{H}\left\|\frac{\Theta^{i}-\Theta^{i-1}}{\tau}\right\|_{H} \\
\leq \tau \frac{1}{2 c_{2}}\left(\theta_{c} c_{\alpha} c_{K}\left(1+\theta_{c}\right)\right)^{2}\left\|\frac{Z^{i}-Z^{i-1}}{\tau}\right\|_{H}^{2}+\tau \frac{c_{2}}{2}\left\|\frac{\Theta^{i}-\Theta^{i-1}}{\tau}\right\|_{H}^{2} \\
\leq \tau \frac{\lambda_{L}+2 \mu_{L} / 3}{2}\left\|\frac{Z^{i}-Z^{i-1}}{\tau}\right\|_{H}^{2}+\tau \frac{c_{2}}{2}\left\|\frac{\Theta^{i}-\Theta^{i-1}}{\tau}\right\|_{H}^{2},
\end{gathered}
$$

so that two terms cancel in (3.22) and we get

$$
\begin{aligned}
& \frac{c_{2}}{4} \tau\left\|\frac{\Theta^{i}-\Theta^{i-1}}{\tau}\right\|_{H}^{2}+\frac{1}{2} \int_{\Omega}\left(\left|\nabla \Theta^{i}\right|^{2}-\left|\nabla \Theta^{i-1}\right|^{2}+\left|\nabla\left(\Theta^{i}-\Theta^{i-1}\right)\right|^{2}\right) \\
& \quad+\tau \frac{C}{4}\left\|\frac{\mathbf{U}^{i}-\mathbf{U}^{i-1}}{\tau}\right\|_{\mathbf{W}}^{2} \leq c \tau \sum_{j=1}^{2}\left(\left\|\frac{\mathcal{X}_{j}^{i}-\mathcal{X}_{j}^{i-1}}{\tau}\right\|_{H}^{2}+\left\|\frac{\mathcal{X}_{j}^{i}-\mathcal{X}_{j}^{i-1}}{\tau}\right\|_{L^{4}(\Omega)}^{4}\right) \\
& \quad+\left\langle\mathcal{R}_{2}^{i}, \Theta^{i}-\Theta^{i-1}\right\rangle+c \tau\left(\left\|\frac{\mathcal{G}^{i}-\mathcal{G}^{i-1}}{\tau}\right\|_{\mathbf{W}^{\prime}}^{2}+\left\|\mathcal{R}_{1}^{i}\right\|_{H}^{2}\right)
\end{aligned}
$$

Finally, by summing up (3.24), for $i=1, \ldots, m$, we write

$$
\begin{aligned}
\frac{c_{2}}{4} \tau \sum_{i=1}^{m} & \left\|\frac{\Theta^{i}-\Theta^{i-1}}{\tau}\right\|_{H}^{2}+\frac{1}{2} \int_{\Omega}\left|\nabla \Theta^{m}\right|^{2}+\tau \sum_{i=1}^{m} \frac{C}{4}\left\|\frac{\mathbf{U}^{i}-\mathbf{U}^{i-1}}{\tau}\right\|_{\mathbf{W}}^{2} \\
\leq & c \tau \sum_{i=1}^{m} \sum_{j=1}^{2}\left(\left\|\frac{\mathcal{X}_{j}^{i}-\mathcal{X}_{j}^{i-1}}{\tau}\right\|_{H}^{2}+\left\|\frac{\mathcal{X}_{j}^{i}-\mathcal{X}_{j}^{i-1}}{\tau}\right\|_{L^{4}(\Omega)}^{4}\right)+\frac{1}{2} \int_{\Omega}\left|\nabla \Theta^{0}\right|^{2} \\
& +\sum_{i=1}^{m}\left|\left\langle\mathcal{R}_{2}^{i}, \Theta^{i}-\Theta^{i-1}\right\rangle\right|+c \tau \sum_{i=1}^{m}\left(\left\|\frac{\mathcal{G}^{i}-\mathcal{G}^{i-1}}{\tau}\right\|_{\mathbf{W}^{\prime}}^{2}+\left\|\mathcal{R}_{1}^{i}\right\|_{H}^{2}\right)
\end{aligned}
$$


We first observe that, on account of $(3.2) \cdots(3.3)$ and (1.27)-(1.28), we can deduce

$$
\begin{aligned}
\tau \sum_{i=1}^{m} & \left(\left\|\frac{\mathcal{G}^{i}-\mathcal{G}^{i-1}}{\tau}\right\|_{\mathbf{W}^{\prime}}^{2}+\left\|\mathcal{R}_{1}^{i}\right\|_{H}^{2}\right) \\
& \leq\|\mathcal{G}\|_{H^{1}\left(0, T ; \mathbf{W}^{\prime}\right)}^{2}+\left\|\mathcal{R}_{1}\right\|_{L^{2}(0 . T ; H)}^{2} .
\end{aligned}
$$

Next, in order to treat the term $\sum_{i=1}^{m}\left|\left\langle\mathcal{R}_{2}^{i}, \Theta^{i}-\Theta^{i-1}\right\rangle\right|$, we exploit a standard procedure and write

$$
\begin{aligned}
& \sum_{i=1}^{m}\left\langle\mathcal{R}_{2}^{i}, \Theta^{i}-\Theta^{i-1}\right\rangle=\left\langle\mathcal{R}_{2}^{m}, \Theta^{m}\right\rangle+\sum_{i=1}^{m-1}\left\langle\mathcal{R}_{2}^{i}-\mathcal{R}_{2}^{i+1}, \Theta^{i}\right\rangle-\left\langle\mathcal{R}_{2}^{1}, \Theta^{0}\right\rangle \\
& \quad \leq\left(2 \max _{1 \leq i \leq m}\left\|\mathcal{R}_{2}^{i}\right\|_{V^{\prime}}+\sum_{i=2}^{m} \tau\left\|\frac{\mathcal{R}_{2}^{i}-\mathcal{R}_{2}^{i-1}}{\tau}\right\|_{V^{\prime}}\right) \max _{0 \leq i \leq m}\left\|\Theta^{i}\right\|_{V} \\
& \quad \leq c\left\|\mathcal{R}_{2}\right\|_{W^{1.1}\left(0, T ; V^{\prime}\right)} \max _{0 \leq i \leq m}\left\|\Theta^{i}\right\|_{V}
\end{aligned}
$$

In addition, we note that the following trivial relation holds:

$$
\left\|\Theta^{m}\right\|_{H}^{2} \leq \tilde{c}\left(\tau^{2} \sum_{i=1}^{m}\left\|\frac{\Theta^{i}-\Theta^{i-1}}{\tau}\right\|_{H}^{2}+\left\|\Theta^{0}\right\|_{H}^{2}\right),
$$

for a suitable constant $\tilde{c}$. Thus, if we apply the Young inequality to (3.27), on account of $(3.28),(3.14)$, and (3.26), from (3.25) we can write

$$
\begin{aligned}
& \max _{1 \leq m \leq \Lambda I}\left\{\frac{c_{2}}{8} \tau \sum_{i=1}^{m}\left\|\frac{\Theta^{i}-\Theta^{i-1}}{\tau}\right\|_{H}^{2}+c_{4}\left\|\Theta^{m}\right\|_{V}^{2}+\tau \sum_{i=1}^{m} \frac{C}{4}\left\|\frac{\mathbf{U}^{i}-\mathbf{U}^{i-1}}{\tau}\right\|_{\mathbf{W}}^{2}\right\} \\
& \leq c+\frac{c_{4}}{2} \max _{0 \leq m \leq M I}\left\|\Theta^{m}\right\|_{V}^{2}+c \tau \sum_{i=1}^{M} \sum_{j=1}^{2}\left\|\frac{\mathcal{X}_{j}^{i}-\mathcal{X}_{j}^{i-1}}{\tau}\right\|_{L^{+}(\Omega)}^{4},
\end{aligned}
$$

for a suitable constant $c_{4}$ and $\tau$ sufficiently small (i.e., we could take $c_{4}=1 / 2$ and $\left.\tau \leq c_{2} /(4 \tilde{c})\right)$, and for any $M$ with $0 \leq M \leq N$. In particular, by (3.29) we can deduce

$$
\begin{gathered}
\tau \sum_{i=1}^{m}\left\|\frac{\Theta^{i}-\Theta^{i-1}}{\tau}\right\|_{H}^{2}+\tau \sum_{i=1}^{m}\left\|\frac{\mathbf{U}^{i}-\mathbf{U}^{i-1}}{\tau}\right\|_{\mathbf{W}}^{2} \\
\leq c+\hat{c} \tau \sum_{i=1}^{m} \sum_{j=1}^{2}\left\|\frac{\mathcal{X}_{j}^{i}-\mathcal{X}_{j}^{i-1}}{\tau}\right\|_{L^{4}(\Omega)}^{4} .
\end{gathered}
$$

for a suitable constant $\hat{c}$ and any integer $m \leq N$.

Now, we would like to apply to (3.29) the discrete Gronwall lemma in the form presented by [14] and establish some uniform bounds on the discrete solutions. To this aim we have to exploit the following a priori estimate in order to control the $L^{4}$-norms in the last term on the right hand side of (3.29).

Fourth A PRIORI estimate. Write (2.16) for $i$ and $i-1, i=2, \ldots, N$, take the difference, and rewrite it in terms of $\left(w_{1}^{i}, w_{2}^{i}\right):=\tau^{-1}\left(\mathcal{X}_{1}^{i}-\mathcal{X}_{1}^{i-1}, \mathcal{X}_{2}^{i}-\mathcal{X}_{2}^{i-1}\right)$. Then, we can 
write

$$
\begin{aligned}
& \left(\begin{array}{c}
w_{1}^{i}-w_{1}^{i-1} \\
w_{2}^{i}-w_{2}^{i-1}
\end{array}\right)+\left(\begin{array}{c}
A\left(w_{1}^{i}-w_{1}^{i-1}\right) \\
A\left(w_{2}^{i}-w_{2}^{i-1}\right)
\end{array}\right)+\tau\left(\begin{array}{l}
A w_{1}^{i} \\
A w_{2}^{i}
\end{array}\right) \\
& +\left(\begin{array}{c}
\Xi_{1}^{i}-\Xi_{1}^{i-1} \\
\Xi_{2}^{i}-\Xi_{2}^{i-1}
\end{array}\right)=\left(\begin{array}{c}
-\lambda\left(\Theta^{i}\right)+\lambda\left(\Theta^{i-1}\right) \\
-\alpha\left(\Theta^{i}\right) Z^{i}+\alpha\left(\Theta^{i-1}\right) Z^{i-1}
\end{array}\right)
\end{aligned}
$$

and test $(3.31)$ by $\left(w_{1}^{i}, w_{2}^{i}\right)$. By similarly proceeding as above, and owing to the Young inequality, we obtain (cf. (2.3), (1.20), (1.19), (1.2), and (3.11))

$$
\begin{aligned}
\frac{1}{2} \sum_{j=1}^{2} & \left(\left\|w_{j}^{i}\right\|_{V}^{2}-\left\|w_{j}^{i-1}\right\|_{V}^{2}+\left\|w_{j}^{i}-w_{j}^{i-1}\right\|_{V}^{2}\right)+\tau \sum_{j=1}^{2} \int_{\Omega}\left|\nabla w_{j}^{i}\right|^{2} \\
\leq & \tau \tilde{c}_{\lambda}\left\|\frac{\Theta^{i}-\Theta^{i-1}}{\tau}\right\|_{H}\left\|w_{1}^{i}\right\|_{H} \\
& +\tau\left(c_{\alpha} \theta_{c} c_{1}\left\|\frac{\Theta^{i}-\Theta^{i-1}}{\tau}\right\|_{H}+\|\alpha\|_{L^{\infty}(\mathbf{R})}\left\|\frac{Z^{i}-Z^{i-1}}{\tau}\right\|_{H}\right)\left\|w_{2}^{i}\right\|_{H} \\
\leq & \delta \tau\left(\left\|\frac{\Theta^{i}-\Theta^{i-1}}{\tau}\right\|_{H}^{2}+\left\|\frac{\mathbf{U}^{i}-\mathbf{U}^{i-1}}{\tau}\right\|_{\mathbf{W}}^{2}\right)+c_{\delta} \tau \sum_{j=1}^{2}\left\|w_{j}^{i}\right\|_{H}^{2},
\end{aligned}
$$

for a suitable $\delta$ we will fix later. Let us point out that, in order to deduce (3.32), we have exploited the following inequality

$$
\sum_{j=1}^{2} \int_{\Omega}\left(\Xi_{j}^{i}-\Xi_{j}^{i-1}\right) w_{j}^{i} \geq 0
$$

holding by monotonicity of the subdifferential (cf. also (2.18)) and the form of the functions $w_{j}^{i}$. Next, we add (3.32) for $i=2, \ldots, m$, with $m \leq N$ and, owing to (3.30), we write

$$
\begin{aligned}
& \frac{1}{2} \sum_{j=1}^{2}\left\|w_{j}^{m}\right\|_{V}^{2} \leq c+\frac{1}{2} \sum_{j=1}^{2}\left\|w_{j}^{1}\right\|_{V}^{2}+c_{\delta} \tau \sum_{j=1}^{2} \sum_{i=1}^{m}\left\|w_{j}^{i}\right\|_{H}^{2} \\
&+\delta \hat{c} \tau \sum_{i=1}^{m} \sum_{j=1}^{2}\left\|\frac{\mathcal{X}_{j}^{i}-\mathcal{X}_{j}^{i-1}}{\tau}\right\|_{L^{4}(\Omega)}^{4} \\
& \leq \frac{1}{2} \sum_{j=1}^{2}\left\|w_{j}^{1}\right\|_{V}^{2}+c_{\delta} \tau \sum_{j=1}^{2} \sum_{i=1}^{m-1}\left\|w_{j}^{i}\right\|_{H}^{2}+c_{\delta} \tau \sum_{j=1}^{2}\left\|w_{j}^{m}\right\|_{H}^{2} \\
&+\delta \hat{c} \tau \sum_{i=1}^{m-1} \sum_{j=1}^{2}\left\|w_{j}^{i}\right\|_{L^{4}(\Omega)}^{4}+\delta \hat{c} \tau \sum_{j=1}^{2}\left\|w_{j}^{m}\right\|_{L^{4}(\Omega)}^{4}+c .
\end{aligned}
$$

Now, we aim to estimate the first term on the right hand side of (3.34). We can proceed directly by writing (2.16) for $i=1$, and test it by $\tau^{-1}\left(\mathcal{X}_{1}^{1}-\mathcal{X}_{1}^{0}, \mathcal{X}_{2}^{1}-\mathcal{X}_{2}^{0}\right)$. Thus, by definition of the subdifferential and (1.30) (cf. also (2.14)), we can test (2.16) written for 
$i=1$ by $\tau^{-1}\left(\mathcal{X}_{1}^{1}-\mathcal{X}_{1}^{0}, \mathcal{X}_{2}^{1}-\mathcal{X}_{2}^{0}\right)$ and write

$$
\begin{aligned}
\sum_{j=1}^{2} \| & \frac{\mathcal{X}_{j}^{1}-\mathcal{X}_{j}^{0}}{\tau} \|_{V}^{2}+\frac{1}{\tau} \int_{\Omega}\left|\nabla\left(\mathcal{X}_{j}^{1}-\mathcal{X}_{j}^{0}\right)\right|^{2} \\
\leq & \|\lambda\|_{L^{\infty}(\mathbf{R})}|\Omega|^{1 / 2}\left\|\frac{\mathcal{X}_{1}^{1}-\mathcal{X}_{1}^{0}}{\tau}\right\|_{H}+\|\alpha\|_{L^{\infty}(\mathbf{R})} c_{1}|\Omega|^{1 / 2}\left\|\frac{\mathcal{X}_{2}^{1}-\mathcal{X}_{2}^{0}}{\tau}\right\|_{H} \\
& +\sum_{j=1}^{2}\left\|A \mathcal{X}_{j}^{0}\right\|_{H}\left\|\frac{\mathcal{X}_{j}^{1}-\mathcal{X}_{j}^{0}}{\tau}\right\|_{H} .
\end{aligned}
$$

Hence, by applying the Young inequality to (3.35), it is easy to deduce a uniform bound for $\left(w_{1}^{1}, w_{2}^{1}\right)$, namely

$$
\left\|w_{j}^{1}\right\|_{V}^{2}=\left\|\frac{\mathcal{X}_{j}^{1}-\mathcal{X}_{j}^{0}}{\tau}\right\|_{V}^{2} \leq c \quad j=1,2 .
$$

Next, by recalling (3.14) and the embedding $H^{1}(\Omega) \hookrightarrow L^{4}(\Omega)$, we can choose $\delta$ such that

$$
\delta \hat{c} \tau \sum_{j=1}^{2}\left\|w_{j}^{m}\right\|_{L^{4}(\Omega)}^{4} \leq \frac{1}{4} \sum_{j=1}^{2}\left\|w_{j}^{m}\right\|_{V}^{2}
$$

and

$$
c_{\delta} \tau \sum_{j=1}^{2}\left\|w_{j}^{m}\right\|_{H}^{2} \leq c
$$

Consequently, by combining (3.36)-(3.38) with (3.34), we finally get

$$
\frac{1}{4} \sum_{j=1}^{2}\left\|w_{j}^{m}\right\|_{V}^{2} \leq c\left(1+\tau \sum_{i=1}^{m-1} \sum_{j=1}^{2}\left\|w_{j}^{i}\right\|_{V}^{2}\left\|w_{j}^{i}\right\|_{V}^{2}+\tau \sum_{i=1}^{m-1} \sum_{j=1}^{2}\left\|w_{j}^{i}\right\|_{H}^{2}\right) .
$$

Hence, we can apply the discrete Gronwall lemma (cf. [14]) to (3.39) and, due to (3.14), deduce

$$
\left\|w_{j}^{m}\right\|_{V}^{2}=\left\|\frac{\mathcal{X}_{j}^{m}-\mathcal{X}_{j}^{m-1}}{\tau}\right\|_{V}^{2} \leq c, \quad j=1,2,
$$

for any $m \leq N$. Now, combining (3.40) with (3.29) yields

$$
\tau \sum_{i=1}^{M}\left\|\frac{\Theta^{i}-\Theta^{i-1}}{\tau}\right\|_{H}^{2}+\max _{1 \leq m \leq M}\left\|\Theta^{m}\right\|_{V}^{2}+\tau \sum_{i=1}^{M}\left\|\frac{\mathbf{U}^{i}-\mathbf{U}^{i-1}}{\tau}\right\|_{\mathbf{W}}^{2} \leq c
$$

for any $M \leq N$.

4. Passage to the limit as $\tau \searrow 0$. In order to prove Theorem 1.1 we aim to pass to the limit as $\tau$ tends to 0 in $(3.5)-(3.7)$. Thus, we can combine the previous estimates 
(3.14), (3.16), (3.40), (3.41), by recalling also (2.3) and (1.2), to obtain, on account of $(3.2)-(3.3)$, and at least for $\tau(0, \hat{\tau})$ for a suitable $\hat{\tau}$,

$$
\begin{aligned}
& \left\|\tilde{\theta}_{\tau}\right\|_{H^{1}(0, T ; H) \cap L^{\infty}(0, T ; V)}+\left\|\theta_{\tau}\right\|_{L^{\infty}(0, T ; V)} \leq c, \\
& \sum_{j=1}^{2}\left\|\tilde{\chi}_{j \tau}\right\|_{W^{1, \infty}(0, T ; V) \cap L^{\infty}\left(0, T ; H^{2}(\Omega)\right)}+\left\|\chi_{j \tau}\right\|_{L^{\infty}\left(0, T ; H^{2}(\Omega)\right)} \leq c, \\
& \left\|\tilde{\mathbf{u}}_{\tau}\right\|_{H^{1}(0, T ; \mathbf{W})}+\left\|\mathbf{u}_{\tau}\right\|_{L^{\infty}(0, T ; \mathbf{W})} \leq c, \\
& \left\|\tilde{z}_{\tau}\right\|_{H^{1}(0, T ; V) \cap L^{\infty}(Q)}+\left\|z_{\tau}\right\|_{L^{\infty}(Q) \cap L^{\infty}(0, T ; V)} \leq c,
\end{aligned}
$$

for $c$ not depending on $\tau \in(0, \hat{\tau})$. Now, it remains to pass to the limit in (3.5)-(3.7) as $\tau \searrow 0$. We first observe that well-known weak and weak star compactness results apply to $(4.1)-(4.4)$ and ensure that the following weak $(\rightarrow)$ and weak star $(\stackrel{*}{\rightarrow})$ convergences hold, at least up to the extraction of suitable subsequences,

$$
\begin{aligned}
& \tilde{\theta}_{\tau} \stackrel{*}{\rightarrow} \theta \text { in } H^{1}(0, T ; H) \cap L^{\infty}(0, T ; V), \\
& \tilde{\chi}_{j \tau} \stackrel{*}{\rightarrow} \chi_{j} \text { in } W^{1, \infty}(0, T ; V) \cap L^{\infty}\left(0, T ; H^{2}(\Omega)\right), \quad j=1,2, \\
& \tilde{\mathbf{u}}_{\tau} \rightarrow \mathbf{u} \text { in } H^{1}(0, T ; \mathbf{W}), \\
& \tilde{z}_{\tau} \stackrel{*}{\rightarrow} z \text { in } H^{1}(0, T ; V) \cap L^{\infty}(Q) .
\end{aligned}
$$

Let us note that, even if you do not specify it, the following convergence results have to be intended to hold up to the extraction of suitable subsequences of $\tau$, still denoted by $\tau$ just for the sake of convenience. Then, owing to strong compactness theorems (cf. [15]), by (4.5), (4.6), and (4.8), we get (actually, something more holds)

$$
\begin{aligned}
& \tilde{\theta}_{\tau} \rightarrow \theta \text { in } C^{0}([0, T] ; H), \\
& \tilde{\chi}_{j \tau} \rightarrow \chi_{j \tau} \text { in } C^{0}([0, T] ; V), \quad j=1,2, \\
& \tilde{z}_{\tau} \rightarrow z \text { in } C^{0}([0, T] ; H),
\end{aligned}
$$

for $z=\operatorname{div} \mathbf{u}$ (cf. (4.7)). Now, since the following relation is fulfilled (cf. (3.2) and (3.3)),

$$
\begin{gathered}
\left\|\tilde{\theta}_{\tau}-\theta_{\tau}\right\|_{L^{\infty}(0, T ; H)}^{2} \leq \max _{1 \leq i \leq N} \tau^{2}\left\|\frac{\Theta^{i}-\Theta^{i-1}}{\tau}\right\|_{H}^{2} \\
\leq \tau\left\|\partial_{t} \tilde{\theta}_{\tau}\right\|_{L^{2}(0, T ; H)}^{2} \leq \tau c,
\end{gathered}
$$

and analogous estimates hold for

$$
\left\|\tilde{\mathbf{u}}_{\tau}-\mathbf{u}_{\tau}\right\|_{L^{\infty}(0, T ; \mathbf{W})}^{2},\left\|\tilde{z}_{\tau}-z_{\tau}\right\|_{L^{\infty}(0, T ; H)}^{2},\left\|\tilde{\chi}_{j \tau}-\chi_{j \tau}\right\|_{L^{\infty}(0, T ; V)}^{2}, \quad j=1,2 .
$$


With the help of (4.5) (4.11) (cf. also (4.1) -(4.4)), we are allowed to infer that

$$
\begin{aligned}
& \theta_{\tau} \rightarrow \theta \text { in } L^{x}(0, T ; H), \quad \theta_{\tau} \stackrel{*}{\rightarrow} \theta \text { in } L^{\infty}(0, T: V), \\
& \chi_{j \tau} \rightarrow \chi_{j} \text { in } L^{\infty}(0, T: V), \\
& \chi_{j \tau} \stackrel{*}{\rightarrow} \chi_{j} \text { in } L^{\infty}\left(0, T: H^{2}(\Omega)\right), \quad j=1,2 . \\
& z_{\tau} \rightarrow \operatorname{div} \mathbf{u} \text { in } L^{\infty}(0, T: H), \\
& z_{\tau} \stackrel{*}{\rightarrow} \operatorname{div} \mathbf{u} \text { in } L^{\infty}(Q) \cap L^{\infty}(0, T: V) . \\
& \mathbf{u}_{\tau} \stackrel{*}{\rightarrow} \mathbf{u} \text { in } L^{\infty}(0, T: \mathbf{W}) .
\end{aligned}
$$

Finally, we observe that it is easily verified (cf. (2.7) - (2.8) and [1])

$$
\begin{aligned}
& \tilde{\mathcal{G}}_{\tau} \rightarrow \mathcal{G} \text { in } L^{2}\left(0, T ; \mathbf{W}^{\prime}\right), \\
& \widetilde{\mathcal{R}}_{2 \tau} \rightarrow \mathcal{R}_{2} \text { in } L^{2}\left(0, T ; V^{\prime}\right), \\
& \mathcal{R}_{1 \tau} \rightarrow \mathcal{R}_{1} \text { in } L^{2}(0, T: H) .
\end{aligned}
$$

Now, by the above convergences, we are in the position of taking the limit as $\tau \searrow 0$ of (3.5)-(3.7). First, we observe that, by (4.16), (4.13)-(4.14), and (4.17), due to (1.20). we can pass to the limit in (3.6) and get (1.17). Let us detail the following convergence results as we apply a procedure we will exploit also in the sequel of our argumentation. Since (4.13)-(4.14) and (1.20) imply, for some subsequence,

$$
\alpha\left(\theta_{\tau}\right) \chi_{2 \tau} \rightarrow \alpha(\theta) \chi_{2} \text { a.e. in } Q .
$$

we owe in addition to (4.2) (cf. (1.11)) and deduce

$$
\left\|\alpha\left(\theta_{\tau}\right) \chi_{2 \tau}\right\|_{L \times(Q)} \leq c .
$$

Then, the Lebesgue dominated convergence theorem yiclds

$$
\alpha\left(\theta_{\tau}\right)_{\chi_{2 \tau}} \rightarrow \alpha(\theta) \chi_{2} \text { in } L^{p}(Q) \text { for any } p<+\infty .
$$

We point out that (4.22) holds for the whole fixed sequence. By arguing as in the previous deduction, on account of (1.20), (1.19), and (4.4) (cf. (2.3)), by (4.13) and (4.15), we obtain

$$
\begin{aligned}
& \lambda\left(\theta_{\tau}\right) \rightarrow \lambda(\theta), \\
& \alpha\left(\theta_{\tau}\right) z_{\tau} \rightarrow \alpha(\theta) \operatorname{div} \mathbf{u}
\end{aligned}
$$

in $L^{p}(Q)$ for any $p<+\infty$. Thus, by virtue of (4.6), (4.14), and (4.23)-(4.24), in order to pass to the limit as $\tau \searrow 0$ in $(3.7)$, it suffices to control the sequence $\left(\xi_{1 \tau}, \xi_{2 \tau}\right)$; i.e., it remains to verify that it converges, in a suitable sense. to some selection $\left(\xi_{1}, \xi_{2}\right)$ of $\partial I_{K . V}\left(\chi_{1}, \chi_{2}\right)$. To this aim. let us observe that, by a comparison in (3.7), (1.19), (4.2). (1.20), and (4.4) imply

$$
\left\|\xi_{j \tau}\right\|_{L^{\infty}\left(0, T ; V^{\prime}\right)} \leq c, \quad j=1,2,
$$

and consequently we have

$$
\xi_{j \tau} \stackrel{*}{\rightarrow} \xi_{j} \text { in } L^{\infty}\left(0, T ; V^{\prime}\right), \quad j=1,2 .
$$


Hence, by (4.14) and (4.26), we can deduce

$$
\sum_{j=1}^{2}\left\langle\xi_{j \tau}, \chi_{j \tau}\right\rangle \rightarrow \sum_{j=1}^{2}\left\langle\xi_{j}, \chi_{j}\right\rangle
$$

as $\tau \searrow 0$, which enables us to apply the result presented in [3, Lemma 1.3, p. 42] and deduce

$$
\left(\xi_{1}, \xi_{2}\right) \in \partial I_{K, V}\left(\chi_{1}, \chi_{2}\right)
$$

which, in particular, yields that $\left(\chi_{1}, \chi_{2}\right) \in K \cap V^{2}$; i.e., the couple $\left(\chi_{1}, \chi_{2}\right)$ satisfies the required constraint (see Remark 1.1). Finally, by the above arguments we can pass to the limit in (3.7) and get (1.16) solved by $\left(\chi_{1}, \chi_{2}\right), \theta$, div $\mathbf{u}$ in $\left(V^{\prime}\right)^{2}$ a.e. in $(0, T)$.

Finally, we aim to pass to the limit in (3.5) and obtain the abstract equation (1.15). To this aim, we first consider the nonlinearities

$$
\begin{aligned}
& S_{1 \tau}=\mathcal{I}_{\tau}\left(F\left(\theta_{\tau}, \chi_{1 \tau}, \chi_{2 \tau}, \mathbf{u}_{\tau}\right)\right), \quad S_{1}=F\left(\theta, \chi_{1}, \chi_{2}, \mathbf{u}\right), \\
& S_{2 \tau}=\mathcal{I}_{\tau}\left(\theta_{\tau} \lambda^{\prime}\left(\theta_{\tau}\right)\right), \quad S_{2}=\theta \lambda^{\prime}(\theta), \\
& S_{3 \tau}=\mathcal{I}_{\tau}\left(\theta_{\tau} \alpha^{\prime}\left(\theta_{\tau}\right) z_{\tau}\right), \quad S_{3}=\theta \alpha^{\prime}(\theta) \operatorname{div} \mathbf{u}, \\
& S_{4 \tau}=\mathcal{I}_{\tau}\left(\theta_{\tau} \alpha^{\prime}\left(\theta_{\tau}\right) \chi_{2 \tau}\right), \quad S_{4}=\theta \alpha^{\prime}(\theta) \chi_{2},
\end{aligned}
$$

and note that by virtue of $(2.3),(1.11),(1.20)$, and (1.19) they are uniformly bounded. Hence, owing to (4.18)-(4.19), (4.5)-(4.6), and (4.8), in order to pass to the limit in $V^{\prime}$ in (3.5), we observe that it suffices to prove that for any $\phi \in L^{2}(0, T ; V)$, there holds

$$
\mathcal{I}_{\tau}\left(S_{j \tau}\right) \phi \rightarrow S_{j} \phi \text { in } L^{2}(Q) .
$$

For the sake of simplicity, let us detail only the case of $S_{1 \tau}$, since the others are mostly similar. Owing to the continuous embedding $H^{1}(\Omega) \hookrightarrow L^{6}(\Omega)$, and by virtue of (4.13)(4.15), (1.19)-(1.20) (cf. also (1.11), and (2.3)), the Lebesgue theorem can be applied to prove that

$$
F\left(\theta_{\tau}, \mathbf{u}_{\tau}, \chi_{1 \tau}, \chi_{2 \tau}\right) \phi \rightarrow F\left(\theta, \mathbf{u}, \chi_{1}, \chi_{2}\right) \phi \text { in } L^{2}(Q), \forall \phi \in L^{2}(0, T ; V),
$$

as $\tau \searrow 0$, and the same convergence easily holds for $\mathcal{I}_{\tau} F\left(\theta_{\tau}, \mathbf{u}_{\tau}, \chi_{1 \tau}, \chi_{2 \tau}\right)$. Indeed, by arguing as in the deduction of (4.22)-(4.24) and recalling (1.20) and (1.19), owing to (4.13) we deduce that $\theta_{\tau} \alpha^{\prime \prime}\left(\theta_{\tau}\right) \rightarrow \theta \alpha^{\prime \prime}(\theta)$ and $\theta_{\tau} \lambda^{\prime \prime}\left(\theta_{\tau}\right) \rightarrow \theta \lambda^{\prime \prime}(\theta)$ in $L^{p}(Q)$ for any $p<$ $+\infty$, as well as an analogous convergence is proved for $z_{\tau} \rightarrow \operatorname{div} \mathbf{u}$ (cf. (2.3) and (4.15)). Hence, by taking, i.e., $p=6$ and by use of (4.14), the generalized Young inequality ensures that (4.31) holds. We can treat by similar arguments the other nonlinearities and we can verify that (4.30) holds for $\mathcal{I}_{\tau}\left(S_{2 \tau}\right), \mathcal{I}_{\tau}\left(S_{3 \tau}\right), \mathcal{I}_{\tau}\left(S_{4 \tau}\right)$ as well. Finally, it remains to verify that

$$
\sum_{j=1}^{2}\left(\partial_{t} \tilde{\chi}_{j \tau}\right)^{2} \rightarrow \sum_{j=1}^{2}\left(\partial_{t} \chi_{j}\right)^{2} \text { in } L^{2}\left(0, T ; V^{\prime}\right) .
$$

Towards this aim, we first note that, thanks to (4.2), we can deduce

$$
\sum_{j=1}^{2}\left\|\left(\partial_{t} \tilde{\chi}_{j \tau}\right)^{2}\right\|_{L^{2}(Q)} \leq c
$$


and consequently, we are allowed to infer that

$$
\left(\partial_{t} \tilde{\chi}_{j \tau}\right)^{2} \rightarrow w_{j} \text { in } L^{2}(Q), \quad j=1,2,
$$

for some subsequence. Thus, in order to show that $w_{j}=\left(\partial_{t} \chi_{j}\right)^{2}, j=1,2$, it suffices to prove that

$$
\partial_{t} \tilde{\chi}_{j \tau} \rightarrow \partial_{t} \chi_{j} \text { in } L^{2}(Q), \quad j=1,2
$$

Indeed, if (4.35) holds, one has

$$
\iint_{Q} \partial_{t} \tilde{\chi}_{j \tau}^{2} \rightarrow \iint_{Q} \partial_{t} \chi_{j}^{2}
$$

while (4.34) implies

$$
\iint_{Q} \partial_{t} \tilde{\chi}_{j \tau}^{2} v \rightarrow \iint_{Q} w_{j} v
$$

for any $v \in L^{2}(Q)$. Thus, by taking $v=1$ in (4.37) and by the uniqueness of the limit of (4.36) we could finally identify $w_{j}$ with $\partial_{t} \chi_{j}^{2}$. First, we observe that (4.6) yields

$$
\sum_{j=1}^{2}\left\|\partial_{t} \chi_{j}\right\|_{L^{2}(Q)}^{2} \leq \liminf _{\tau \searrow 0} \sum_{j=1}^{2}\left\|\partial_{t} \tilde{\chi}_{j \tau}\right\|_{L^{2}(Q)}^{2},
$$

and consequently (4.35) can be obtained just by verifying that

$$
\underset{\tau \searrow 0}{\limsup } \sum_{j=1}^{2}\left\|\partial_{t} \tilde{\chi}_{j \tau}\right\|_{L^{2}(Q)}^{2} \leq \sum_{j=1}^{2}\left\|\partial_{t} \chi_{j}\right\|_{L^{2}(Q)}^{2},
$$

since the convergence of the norms combined with the weak convergence of the sequence imply the required strong convergence (4.35). To this aim, we test (3.7) by $\left(\partial_{t} \tilde{\chi}_{1 \tau}, \partial_{t} \tilde{\chi}_{2 \tau}\right)$, integrate over $(0, T)$, and then take the limsup as $\tau \searrow 0$. We have

$$
\begin{aligned}
\underset{\tau \searrow 0}{\limsup } \sum_{j=1}^{2}\left\|\partial_{t} \tilde{\chi}_{j \tau}\right\|_{L^{2}(Q)}^{2} \\
=\limsup _{\tau \searrow 0}\left(\sum _ { j = 1 } ^ { 2 } \left(-\left\|\nabla\left(\partial_{t} \tilde{\chi}_{j \tau}\right)\right\|_{L^{2}(Q)}^{2}-\int_{0}^{T} \int_{\Omega} \nabla \chi_{j \tau} \nabla\left(\partial_{t} \tilde{\chi}_{j \tau}\right)\right.\right. \\
\left.\left.\quad-\int_{0}^{T}\left\langle\xi_{j \tau}, \partial_{t} \tilde{\chi}_{j \tau}\right\rangle\right)-\int_{0}^{T} \int_{\Omega} \lambda\left(\theta_{\tau}\right) \partial_{t} \tilde{\chi}_{1 \tau}-\int_{0}^{T} \int_{\Omega} \alpha\left(\theta_{\tau}\right) z_{\tau} \partial_{t} \tilde{\chi}_{2 \tau}\right) .
\end{aligned}
$$

We first note that, since $(1.20),(1.19),(4.13)-(4.15)$, and (4.6) hold, we can infer that (cf. also (4.23)-(4.24))

$$
\begin{aligned}
\lim _{\tau \searrow 0} & \sum_{j=1}^{2}-\int_{0}^{T} \int_{\Omega} \nabla \chi_{j \tau} \nabla\left(\partial_{t} \tilde{\chi}_{j \tau}\right)-\int_{0}^{T} \int_{\Omega} \lambda\left(\theta_{\tau}\right) \partial_{t} \tilde{\chi}_{1 \tau} \\
& -\int_{0}^{T} \int_{\Omega} \alpha\left(\theta_{\tau}\right) z_{\tau} \partial_{t} \tilde{\chi}_{2 \tau}=\sum_{j=1}^{2}-\int_{0}^{T} \int_{\Omega} \nabla \chi_{j} \nabla\left(\chi_{j t}\right) \\
& -\int_{0}^{T} \int_{\Omega} \lambda(\theta) \chi_{1 t}-\int_{0}^{T} \int_{\Omega} \alpha(\theta) \operatorname{div} \mathbf{u} \chi_{2 t} .
\end{aligned}
$$


Moreover, owing to (4.6), by the weak lower semicontinuity of the norm, we have

$$
\begin{aligned}
& \limsup _{\tau \searrow 0} \sum_{j=1}^{2}\left(-\left\|\nabla\left(\partial_{t} \tilde{\chi}_{j \tau}\right)\right\|_{L^{2}(Q)}^{2}\right) \\
& \quad=-\liminf _{\tau \searrow 0} \sum_{j=1}^{2}\left\|\nabla\left(\partial_{t} \tilde{\chi}_{j \tau}\right)\right\|_{L^{2}(Q)}^{2} \leq \sum_{j=1}^{2}-\left\|\nabla\left(\partial_{t} \chi_{j}\right)\right\|_{L^{2}(Q)}^{2} .
\end{aligned}
$$

Finally, we have to treat the term $\sum_{j=1}^{2}-\int_{0}^{T}\left\langle\xi_{j \tau}, \partial_{t} \tilde{\chi}_{j \tau}\right\rangle$. To this aim, we observe that by definition of the subdifferential and owing to (3.2)-(3.3), we can write (cf. [3])

$$
\begin{aligned}
& \sum_{j=1}^{2} \int_{0}^{T}\left\langle\xi_{j \tau}, \partial_{t} \tilde{\chi}_{j \tau}\right\rangle=\sum_{j=1}^{2} \tau \sum_{i=1}^{N}\left\langle\Xi_{j}^{i}, \frac{\mathcal{X}_{j}^{i}-\mathcal{X}_{j}^{i-1}}{\tau}\right\rangle \\
& \quad=\sum_{j=1}^{2} \sum_{i=1}^{N}\left\langle\Xi_{j}^{i}, \mathcal{X}_{j}^{i}-\mathcal{X}_{j}^{i-1}\right\rangle \geq \sum_{i=1}^{N}\left(I_{K \cap V^{2}}\left(\mathcal{X}_{1}^{i}, \mathcal{X}_{2}^{i}\right)-I_{K \cap V^{2}}\left(\mathcal{X}_{1}^{i-1}, \mathcal{X}_{2}^{i-1}\right)\right) \\
& \quad=I_{K \cap V^{2}}\left(\mathcal{X}_{1}^{N}, \mathcal{X}_{2}^{N}\right)-I_{K \cap V^{2}}\left(\mathcal{X}_{1}^{0}, \mathcal{X}_{2}^{0}\right) \\
& \quad=I_{K \cap V^{2}}\left(\tilde{\chi}_{1 \tau}(T), \tilde{\chi}_{2 \tau}(T)\right)-I_{K \cap V^{2}}\left(\chi_{10}, \chi_{20}\right)
\end{aligned}
$$

for any $\tau>0$. Hence, by the lower semicontinuity in $V$ of the function $I_{K \cap V^{2}}$ and due to $(4.10)$, we claim

$$
\begin{aligned}
\underset{\tau \searrow 0}{\limsup } & -\sum_{j=1}^{2} \int_{0}^{T}\left\langle\xi_{j \tau}, \partial_{t} \tilde{\chi}_{j \tau}\right\rangle=-\liminf _{\tau \searrow 0} \sum_{j=1}^{2} \int_{0}^{T}\left\langle\xi_{j \tau}, \partial_{t} \tilde{\chi}_{j \tau}\right\rangle \\
& \leq-\liminf _{\tau \searrow 0}\left(I_{K \cap V^{2}}\left(\tilde{\chi}_{1 \tau}(T), \tilde{\chi}_{2 \tau}(T)\right)-I_{K \cap V^{2}}\left(\chi_{10}, \chi_{20}\right)\right) \\
& \leq-I_{K \cap V^{2}}\left(\chi_{1}(T), \chi_{2}(T)\right)+I_{K \cap V^{2}}\left(\chi_{10}, \chi_{20}\right) .
\end{aligned}
$$

Now, by combining (4.40), (4.41), and (4.43), we get

$$
\begin{aligned}
\underset{\tau \searrow 0}{\limsup } & \sum_{j=1}^{2}\left\|\partial_{t} \tilde{\chi}_{j \tau}\right\|_{L^{2}(Q)}^{2} \leq \sum_{j=1}^{2}\left(-\int_{0}^{T} \int_{\Omega} \nabla \chi_{j} \nabla\left(\chi_{j t}\right)-\left\|\nabla\left(\chi_{j t}\right)\right\|_{L^{2}(Q)}^{2}\right) \\
& -\int_{0}^{T} \int_{\Omega} \lambda(\theta) \chi_{1 t}-\int_{0}^{T} \int_{\Omega} \alpha(\theta) \operatorname{div} \mathbf{u} \chi_{2 t} \\
& -I_{K \cap V^{2}}\left(\chi_{1}(T), \chi_{2}(T)\right)+I_{K \cap V^{2}}\left(\chi_{10}, \chi_{20}\right),
\end{aligned}
$$

and the right hand side of (4.44) is equal to

$$
\sum_{j=1}^{2}\left\|\chi_{j t}\right\|_{L^{2}(Q)}^{2}
$$

as one easily verifies by testing $(1.16)$ by $\left(\chi_{1 t}, \chi_{2 t}\right)$ and then integrating in time (see also the following remark).

REMARK 4.1. The last result easily follows once it is proved that

$$
-I_{K \cap V^{2}}\left(\chi_{1}(T), \chi_{2}(T)\right)+I_{K \cap V^{2}}\left(\chi_{10}, \chi_{20}\right)=-\sum_{i=1}^{2} \int_{0}^{T}\left\langle\xi_{i}, \chi_{i t}\right\rangle,
$$


and this can be obtained by extending the statement of Lemma 3.3, p. 73, in [4] to the case of abstract subdifferential operators defined in the duality pairing between $\left(V^{\prime}\right)^{2}$ and $V^{2}$.

\section{REFERENCES}

[1] S. Aizicovici, P. Colli, and M. Grasselli. Doubly nonlinear evolution equations with memory, Funkcial. Ekvac. 44, 19-51 (2001)

[2] C. Baiocchi, Sulle equazioni differenziali astratte del primo e del secondo ordine negli spazi di Hilbert, Ann. Mat. Pura Appl. 76(4), 233--304 (1967)

[3] V. Barbu, Nonlinear semigroups and differential equations in Banach spaces, Noordhoff, Leyden, 1976

[4] H. Brézis, Opérateurs maximaux monotones et semi-groups de contractions dans les espaces de Hilbert, North-Holland Math. Stud. 5, North-Holland, Amsterdam, 1973

[5] E. Bonetti, Global solvability of a dissipative Frémond model for shape memory alloys. Part I: mathematical formulation and uniqueness, Quart. Appl. Math. 61(4), 759-781 (2003)

[6] E. Bonetti, Global solution to a Frémond model for shape memory alloys with thermal memory, Nonlinear Anal. 46, 535-565 (2001)

[7] N. Chemetov, Uniqueness results for the full Frémond model of shape memory alloys, Z. Anal. Anwendungen 17, 877-892 (1998)

[8] P. Colli, Global existence for the three-dimensional Frémond model of shape memory alloys, Nonlinear Anal. 24, 1565-1579 (1995)

[9] P. Colli, M. Frémond, and A. Visintin, Thermo-mechanical evolution of shape memory alloys, Quart. Appl. Math. 48, 31-47 (1990)

[10] M. Frémond, Matériaux a mémoire de forme, C. R. Acad. Sci. Paris Sér. II Méc. Phys. Chim. Sci. Univers. Sci. Terre. 304, 239-244 (1987)

[11] M. Frémond, Shape memory alloys. A thermomechanical model, in: Free Boundary Problems: Theory and applications, vol. I-II (K. H. Hoffmann and J. Sprekels, eds.), Pitman Res. Notes Math. Ser. 185, Longman, London, 1990

[12] M. Frémond, Non smooth thermo-mechanics, Springer-Verlag, 2001

[13] M. Frémond and S. Miyazaki, Shape memory alloys, in: CISM Courses and Lectures No. 351, Springer-Verlag, New York, 1996

[14] J. W. Jerome, Approximation of nonlinear evolution systems, in: Ser. Math. in Sci. and Eng., 164, Academic Press, 1983

[15] J. Simon, Compact sets in the space $L^{p}(0, T ; B)$, Ann. Mat. Pura Appl. 146(4), 65-96 (1987)

[16] J. Sprekels, Shape memory alloys: mathematical models for a class of first order solid-solid phase transitions in metals, Control Cybernet. 19, 287. 308 (1990) 